\title{
Characterisation of sheet cavity noise of a hydrofoil using the Ffowcs Williams-Hawkings acoustic analogy
}

\author{
Artur K. Lidtke ${ }^{\mathrm{a}, *}$, Stephen R. Turnock ${ }^{\mathrm{a}}$, Victor F. Humphrey ${ }^{\mathrm{b}}$ \\ ${ }^{a}$ Fluid Structure Interactions Group, University of Southampton, SO16 7QF, UK \\ ${ }^{b}$ Institute of Sound and Vibration Research, University of Southampton, SO17 1BJ, UK
}

\begin{abstract}
Concerns about pollution of the marine environment with ship-induced noise and the scarcity of available numerical methods have recently stimulated significant amounts of research in hydroacoustic modelling. In this work, Large Eddy Simulation (LES) is used with Schnerr-Sauer mass- transfer cavitation model and a porous Ffowcs WilliamsHawkings (FW-H) acoustic analogy in order to simulate sheet cavitation on a NACA0009 hydrofoil. The aim is to investigate how well the proposed method captures the dominant noise sources associated with periodic sheet cavitation. The study further focuses on practical aspects, such as the importance of the non-linear FW-H term and convergence of the acoustic solution depending on the choice of the integration surface. This is done by correlating the radiated noise with integral and local flow quantities, such as cavity volume, lift coefficient and local vapour content. A key finding of the study is that the simulation framework is capable of correctly capturing the monopole nature of the sound generated by an oscillating cavity sheet. Results indicate that the numerical method is incapable of accurately resolving the flow during the final collapse stages of smaller cavity clouds, mainly due to mesh density limitations and the use of an incompressible flow assumption. Lack of small-scale bubble structures also causes the high-frequency range of the noise spectra to be under-predicted. Despite certain limitations the presented method offers a significant insight into the nature of cavitation-dominated noise and allows for some of the dominant sound generating mechanisms to be categorised.
\end{abstract}

Keywords: Hydroacoustics, CFD, Cavitation, Large Eddy Simulation, Acoustic analogy, Hydrofoil

\section{Introduction}

In recent years concerns have been raised about the effect of persistent noise, such as that present near the shipping lanes, on the marine environment, and uncertainty associated with how or whether this should be regulated in the future [1]. This has driven an increased interest in studying the noise signature of commercial vessels, resulting in projects such as SONIC or AQUO $[2,3]$. Currently, expensive full-scale measurements constitute the most accurate way of quantifying the noise emitted by a single ship, with model-scale experimental tests being subject to multiple scaling assumptions [4]. It is, therefore, important to develop computational means of computing the noise signature of a marine propeller in order to better address the aforementioned concerns.

\footnotetext{
${ }^{*}$ Corresponding author.

Email address: akl1g09@soton.ac.uk (Artur K. Lidtke )
}

A ship propulsor operates in a highly non-uniform and turbulent wake, which causes periodic and nonperiodic pressure fluctuations and hence noise $[5,6,7$, 4]. Sound emitted by a marine propeller is also affected by the occurrence of cavitation, which happens due to expansion of small gas nuclei present in the liquid when they are subject to tensile stress in low pressure regions [8]. Close to the design conditions a well designed propeller will typically not experience significant amounts of cavitation, some is still likely to be present if high efficiency, associated with high blade loading, is to be achieved $[9,10,11]$. The unsteady inflow, ship motions and presence of a wake may also induce additional cavitation mechanisms by causing loading variations [12]. These features make numerically capturing marine propeller noise a challenging problem.

In order to compute an acoustic disturbance one has to consider a fluid in a compressible form. Although several applications using such solvers for cavitation modelling have been reported in the literature 
$[13,14,15]$, the small time step requirements imposed by the speed of sound in water may limit the applicability of such simulations from an engineering perspective [16]. Furthermore, if radiated sound is to be considered, rather than the near-field hydrodynamic fluctuations, one would be required to solve the flow in an exceedingly large domain with high mesh density [17]. One of the alternatives is the use of acoustic analogies, such as that formulated by Ffowcs Williams-Hawkings (FW-H) [18]. This aims to use the near-field flow data for a control surface, located either on or around the body moving through fluid, in order to predict how the sound generated will radiate out into the far-field, as depicted in Figure 1.

Few detailed studies using the Ffowcs WilliamsHawkings analogy and advanced flow modelling techniques to investigate the cavitation induced noise have been presented in the literature to date. Given the limited discussion, it is necessary to gain further insight into how well this branch of acoustic analogies performs when used with the most widely used cavitation models before further studies looking at ship-induced noise may be pursued. Specifically, a better understanding of the importance of the incompressible flow assumption, the influence of the level of complexity of turbulence modelling, the effect of the cavitation model assumptions, the dependence of the acoustic solution on the choice of the integration surface, and the relative importance of specific FW-H terms needs to be developed.

In order to address these questions, the present study has investigated the flow around a hydrofoil representing the modelling challenges associated with cavitation noise modelling on a ship propeller. The porous Ffowcs Williams-Hawkings analogy [18, 19] implemented in OpenFOAM, the cavitation model of Sauer \& Schnerr [20], and incompressible Large Eddy Simulation (LES) based on the hybrid dynamic-mixed Smagorinsky model were used to provide the hydrodynamic and hydroacoustic solutions. Focus of the analysis was put on analysing the relationships between the observed flow features and the radiated sound, indicating the potential advantages and disadvantages of the presented methodology.

\section{Theory}

\subsection{Cavitation modelling}

Figure 1 illustrates main cavitation mechanisms occurring around a hydrofoil and generating noise. Firstly, large cavities, such as cavity sheets, act to modify the path of the flow, often changing the effective profile of the foil section [21]. As their volume fluctuates, a lowfrequency, monopole noise source is created [11, 22]. Secondly, large amounts of smaller bubbles of varying sizes are often present in cavitating flow [23, 24, 9]. As these travel through regions of changing pressure they may become excited and their size may oscillate, also generating a monopole noise term. Given the small size of these bubbles their oscillation frequencies are typically higher than those experienced by larger cavities and may be over a kilohertz [25]. Finally, the collapse of cavities generates high-amplitude noise peaks [26]. This behaviour is rapid, leads to high interface velocities, and is affected by factors such as non-condensible gas distribution, compressibility effects, surface tension, and thermodynamic properties of the fluids involved [8].

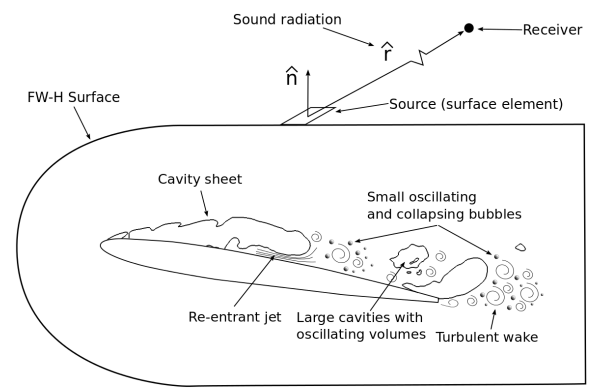

Figure 1: Illustration of the dominant flow and cavitation features present on a hydrofoil and how they are related to the radiated noise computed using the acoustic analogy.

Several main categories of approaches to modelling cavitation exist, ranging from the simpler boundary element methods to detailed bubble-tracking, or Lagrangian, methods [21, 24, 23]. The use of these in practical applications is limited either by their simplicity or high computational cost. Alternatively, the multiphase problem may be modelled using single-fluid methods. Such models rely on techniques such as volume of fluid (VOF) in order to resolve the multiphase flow on an $\mathrm{Eu}-$ lerian grid. A difficulty in using this modelling technique is keeping the interface of the two fluids sharp and in its reconstruction.

The Schnerr-Sauer cavitation model was used [20]. Its accuracy in terms of cavity extents and unsteady behaviour has been demonstrated in previously reported numerical investigations [27, 28, 29]. Moreover, the model is not dependent on any empirical coefficients, per se, although relies on the choice of the water quality properties, namely nuclei density, $n_{0}$, and mean nuclei radius, $R_{0}$. It also allows standard turbulence modelling 
techniques, such as Reynolds Averaged Navier Stokes or Large Eddy Simulation, to be used without any further modifications $[27,30]$.

In this approach, the volume fraction of liquid is governed by a scalar transport equation,

$$
\frac{\partial \alpha}{\partial t}+\nabla \cdot(\alpha \mathbf{U})=-\frac{\dot{m}}{\rho},
$$

where $\alpha$ is the volume fraction, $\mathbf{U}$ and $\rho$ are the flow velocity and density, respectively, and $\dot{m}$ is a source term representing the rate of transfer of mass between the liquid and vapour phases induced by cavitation. Fluid density and viscosity, $\rho$ and $\mu$, are interpolated in accordance with the mass conservative, immiscible fluid mixture assumption of the VOF method,

$$
\phi=\alpha \phi_{\text {liquid }}+(1-\alpha) \phi_{\text {vapour }}
$$

The pressure equation is also modified by introduction of the $\dot{m}$ source term in order to account for a velocity divergence induced by the mass transfer, yielding

$$
\nabla \cdot \overline{\mathbf{U}}=\left(\frac{1}{\rho_{v}}-\frac{1}{\rho_{l}}\right) \dot{m}
$$

The role of the cavitation model is to account for the net rate of mass being transferred between the liquid and vapour phases, $\dot{m}$ in (1), or, in other words, predict the phase change from liquid to vapour (vaporisation) and vice versa (condensation). The used model approximates the behaviour of individual bubbles present in the fluid and being governed by the Rayleigh-Plesset equation $[20,8]$ and computes their equivalent mass transfer rate as

$$
\dot{m}=C \frac{\rho_{\mathrm{l}} \rho_{\mathrm{v}}}{\rho}(1-\alpha) \alpha \frac{3}{R} \sqrt{\frac{\frac{2}{3}\left(p-p_{\mathrm{v}}\right)}{\rho_{\mathrm{l}}}},
$$

where the radius $R$ is modelled based on $R_{0}$ and $n_{0}, C$ is a constant tuning parameter set to 1.0 not to artificially alter the cavitation behaviour. In the OpenFOAM 2.2.2 implementation the mass transfer source term is split into condensation and vaporisation terms based on the sign of $p-p_{v}$.

\subsection{Acoustic analogy}

Acoustic analogies have been widely used for aeroacoustic noise characterisation $[31,32,33]$. Among the research related to marine propeller noise is the work by Ianniello et al. [7] who investigated the noise induced by a full scale ship in non-cavitating conditions using a porous FW-H analogy coupled with a RANS solver. Pan \& Zhang [34] studied the non-cavitating noise for the DTMB 4118 propeller in non-uniform inflow conditions using FW-H analogy evaluated on the propeller blade and a Detached Eddy Simulation (DES) flow solver. Studies by Seol et al. [11] and Salvatore \& Ianniello [21] both described using a potential flow solver with a FW-H analogy evaluated on the blade surface to compute the noise of a propeller in cavitating and non-cavitating conditions. More authors have recently presented initial results involving the use of acoustic analogies for the assessment of noise signature of various ships [35, 36, 37].

The Ffowcs-Williams Hawkings acoustic analogy uses the extended Lighthill's equation to predict noise when moving surfaces are present [18].It may be derived by rearrangement of mass and momentum conservation equations in order to yield an inhomogeneous wave equation $[18,38]$. Its solution may be expressed as a summation of three different terms yielding the total pressure disturbance at the location of a receiver

$$
p^{\prime}(\mathbf{x}, t)=p_{T}^{\prime}(\mathbf{x}, t)+p_{L}^{\prime}(\mathbf{x}, t)+p_{Q}^{\prime}(\mathbf{x}, t) .
$$

In the above $(\mathbf{x}, t)$ is the receiver location and time, $p$ 'denotes the acoustic pressure disturbance and its individual elements are denoted with subscripts referring to: $T$ - thickness, $L$ - loading, and $Q$ - non-linear contributions [21, 38]. The first two of the terms in (5) are computed using a surface integral, while $p_{Q}^{\prime}$ is represented by a volume integral. The classical version of the analogy evaluates the $p_{T}^{\prime}$ and $p_{L}^{\prime}$ terms of the equation on the surface of the body.

In order to better account for noise sources located off the geometry, such as those induced by cavitation or turbulent wake structures, this study makes use of the porous formulation, formally derived by Di Francescantonio [19]. In this approach the integration surface is defined in such way as to enclose both the body moving through fluid and a volume around it. This imposes certain needs on the spatial resolution of the mesh enclosed by the surface in order to avoid dissipation and dispersion errors but allows noise sources present inside the flow, such as those due to detached cavitation, to be accounted for. For such an approach terms $p_{T}^{\prime}$ and $p_{L}^{\prime}$ in Equation (5) may no longer be interpreted as thickness or loading contributions as they no longer represent physical sources of a given order. In this work they shall be referred to as pseudo-thickness and pseudoloading, respectively. A schematic representation of how a porous surface is defined relative to the flow is given in Figure 1.

Before further considering the $\mathrm{FW}-\mathrm{H}$ equations it is useful to define intermediate acoustic variables, which 
for a stationary control surface may be expressed as

$$
\begin{aligned}
U_{i} & =\frac{\rho}{\rho_{0}} u_{i}, \\
L_{i} & =P_{i j} \hat{n}_{j}+\rho u_{i}\left(u_{i} \cdot \hat{n}_{i}\right),
\end{aligned}
$$

where $u$ is the flow velocity and $P_{i j}$ is the compressive stress tensor. Given the pressure field of a fluid, $p$, and its value when the fluid is at rest, $p_{0}$, one may simplify the $P_{i j}$ tensor to $p-p_{0}[21,38]$. For compressible flow the fluid density is composed of a steady value, $\rho_{0}$ and a density disturbance, $\rho^{\prime}$, yielding $\rho=\rho^{\prime}+\rho_{0}$.

For the case of a stationary control surface both the receiver, $\mathbf{x}$, and source, $\mathbf{y}$, positions become constant and the Mach number of the control surface becomes zero. This allows the surface integrals $p_{T}^{\prime}$ and $p_{L}^{\prime}$ from (5) to be written as

$$
\begin{aligned}
& 4 \pi p_{T}^{\prime}(\mathbf{x}, t)=\frac{\partial}{\partial t} \int_{S}\left[\frac{\rho_{0} U_{n}}{r}\right]_{\tau} \mathrm{d} S, \\
& 4 \pi p_{L}^{\prime}(\mathbf{x}, t)=\frac{1}{c_{0}} \frac{\partial}{\partial t} \int_{S}\left[\frac{L_{r}}{r}\right]_{\tau} \mathrm{d} S+\int_{S}\left[\frac{L_{r}}{r^{2}}\right]_{\tau} \mathrm{d} S .
\end{aligned}
$$

where $c_{0}$ is the speed of sound. Projections in sourceobserver or surface normal directions are indicated by subscripts $r$ or $n$, respectively, for instance in $U_{n}$. $\int_{S}[\ldots] \mathrm{d} S$ denotes integration over the porous surface. Each of the terms in (7) is evaluated at the retarded time, denoted by $[\ldots]_{\tau}$. This accounts for the sound propagating from different elements of the discretised control surface taking a finite time to reach the receiver $[39,38,21,17]$. Thus the contribution of an individual surface element located at $\mathbf{y}$ at the emission (retarded) time $\tau$ will affect the receiver pressure at time

$$
t=\tau+\frac{|\mathbf{x}(t)-\mathbf{y}(\tau)|}{c} .
$$

From a practical standpoint this requires the acoustic pressure signals to be interpolated onto a coherent time grid given a unique $t$ value for each face-receiver combination. In the present method this is done by extending the CFD time axis using a fixed time step and linearly extrapolating the acoustic contribution of a given face onto the time indices closest to its analytically computed listener time.

$p_{Q}^{\prime}(\mathbf{x}, t)$ in (5) is the contribution from non-linear sources and should be accounted for using a volume integral. For a porous formulation this only applies to sources located outside of the integration surface $[31,7]$. This means that $p_{Q}^{\prime}(\mathbf{x}, t) \rightarrow 0$ in a numerical sense if the control surface is assumed to enclose all relevant noise sources. Thus Equations (7a) and (7b) should, in theory, provide a complete solution to the original Equation (5). This emphasises the importance of the control surface having to enclose the entire region of interest and highlights a potential source of errors associated with user inputs at the pre-processing stage. From a practical standpoint it may not be trivial to decide how large a control surface is needed for the errors associated with neglecting $p_{Q}^{\prime}(\mathbf{x}, t)$ to be small. Hence the convergence of the acoustic solution depending on the choice of the FW-H surface is discussed later for the hydrofoil case study considered.

The acoustic analogy was implemented as a runtime and stand-alone post-processing utility based on the OpenFOAM 2.2.2 C++ libraries. This allows for the acoustics module to be used with arbitrary flow solvers provided in OpenFOAM without the need of any external data transfer. The control surface is constructed from a set of mesh faces, which removes the need for additional interpolation that could potentially have a detrimental effect on capturing small-scale perturbations. The downside of this choice is the limited control over the integration surface discretisation if the flow solution is to remain unaffected. Steady-state pressure field is supplied from RANS results used to initialise the LES simulation.

\subsection{Acoustic analogy validation}

A set of simulations was carried out whereby analytical acoustic monopoles and dipoles were used to to provide the flow-field data. Figure 2 shows an example of the time history of the pressure signal for a receiver located $1 \mathrm{~m}$ away from a monopole source of strength chosen so that the listener pressure varies between $\pm 1 P a$. The analytical data is computed using

$$
p^{\prime}(\mathbf{x}, t)=\frac{j \omega \rho Q e^{j(\omega t-k|\mathbf{x}-\mathbf{y}|)}}{4 \pi|\mathbf{x}-\mathbf{y}|},
$$

where $\mathbf{y}$ is the location of the source, $\mathbf{x}$ is the point at which the sound pressure is being evaluated, $k$ is the wave number, $\omega$ the frequency of oscillation, and $Q$ is the strength of the source describing the amount of volume flow it induces [40].

Accounting for the retarded time eliminates the phase error between the analytical and predicted pressure at the receiver. An initial jump in the predicted signal associated with stabilisation of the finite derivatives is visible in the data. Overall, good agreement is seen between the predicted and analytical signals.

\subsection{Large Eddy Simulation}

Large Eddy Simulation was used as it allowed unsteady features associated with cavitation dynamics to 


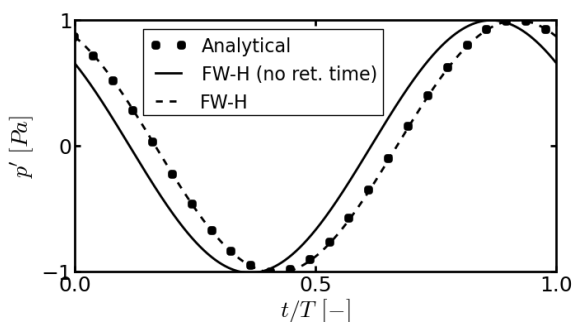

Figure 2: Comparison of the analytical and predicted pressure signal induced by a monopole source showing the effect of the retarded time.

be predicted more accurately. This is because simpler methods, such as URANS, are prone to added numerical diffusion and thus under-predict unsteady flow features. Moreover, the rapid changes experienced by the flow may violate the core RANS assumptions. The VOF multiphase approach requires a fine grid resolution in order to provide sufficient accuracy, irrespective of the turbulence modelling technique used. This implies that the difference in computational effort between URANS and LES is expected to be comparably small and so the latter may seem more appealing than for a non-cavitating flow simulation, given the added accuracy [16].

LES involves solving the filtered Navier-Stokes equations. Motions of large scales are fully resolved and the ones smaller than the grid resolution are modelled. Governing equations of motion are solved using the finite volume method using Pressure implicit with splitting of operator (PISO) algorithm supplied by OpenFOAM. Filtering of the equations is done by multiplying the quantity in question with a convolution operator whose kernel is defined by the filter width related to the mesh size. By denoting the filtering operation with an overbar, the incompressible forms of momentum and continuity equations become

$$
\begin{aligned}
\frac{\partial \overline{\mathbf{U}}}{\partial t}+\nabla \cdot(\overline{\mathbf{U}} \otimes \overline{\mathbf{U}}) & =-\frac{1}{\rho} \nabla \bar{p}+v \nabla^{2} \overline{\mathbf{U}}-\nabla \cdot \tau, \\
\nabla \cdot \overline{\mathbf{U}} & =0,
\end{aligned}
$$

where $\tau$ is the non-linear subgrid stress tensor,

$$
\tau=\overline{\mathbf{U} \otimes \mathbf{U}}-\overline{\mathbf{U}} \otimes \overline{\mathbf{U}} .
$$

Dynamic mixed Smagorisnky model, combing the scale similarity hypothesis-based model by Bardina [41] with Smagorinsky model derived from the Boussinesq hypothesis, was used. Following Zang et al. [42] this may be expressed as

$$
\tau^{S}-\frac{1}{3} \tau^{S} \cdot \mathbf{I}=\frac{1}{2}\left(-2 v_{\mathrm{SGS}} \overline{\mathbf{S}}+\tau^{B}-\frac{1}{3} \tau^{B} \mathbf{I}\right)
$$

In the above superscripts $S$ and $B$ denote the Smagorinsky and Bardina model contributions. $\mathbf{S}$ is the fluid strain rate and the assumed subgrid scale viscosity is denoted for a filter width, $\Delta$, as $v_{\mathrm{SGS}}=\left(C_{S} \bar{\Delta}\right)^{2}|\mathbf{S}|$. Cube root volume was used as the filter length and the model $C_{e}$ coefficient was set to 1.048 [6]. Dynamic Smagorinsky constant was modelled using [43]

$$
C_{\mathrm{S}}^{2}=\frac{1}{2}\left(\frac{(\mathbf{L}-\mathbf{B}) \cdot \mathbf{M}}{\mathbf{M} \cdot \mathbf{M}}\right),
$$

where

$$
\begin{aligned}
& \mathbf{M}=2(\bar{\Delta} / \hat{\Delta})^{2}|\hat{\mathbf{S}}| \hat{\mathbf{S}}-\hat{\Delta}^{2} \mid \widehat{\overline{\mathbf{S}} \mid \overline{\mathbf{S}}}, \\
& \mathbf{B}=\widehat{\overline{\overline{\mathbf{U}}} \otimes \overline{\overline{\mathbf{U}}}-\hat{\overline{\mathbf{U}}} \otimes \hat{\overline{\mathbf{U}}}} \\
& \mathbf{L}=\widehat{\overline{\mathbf{U}} \otimes \overline{\mathbf{U}}}-\hat{\overline{\mathbf{U}}} \otimes \hat{\mathbf{U}} .
\end{aligned}
$$

\subsection{LES model validation}

The used subgrid-scale model has been subject to validation in the study by Lloyd [6], where it was applied both to canonical channel flows as well as hydroacoustic analysis of foil sections and tidal turbines at comparable Reynolds numbers to the case considered here. To further test the model, flow over a NACA0012 section at Reynolds number of 1.5 million and at an angle of attack of $6^{\circ}$ was computed, using the same grid topology, boundary conditions and wall treatment as in the present study. The total cell count used was 5.6 million. The results showed that the inaccuracies introduced by the wall function have a similar effect on the integral flow quantities as in the case of a steady RANS simulation, as shown in Table 1. Given the reduction in the computational time offered by the wall function and the fact that the flow features of primary interest were expected to be away from the body surface this was deemed satisfactory for the purpose of this study.

\section{Method}

\subsection{Test case description}

A symmetric NACA0009 foil section was studied, continuing the previous studies presented by the authors [44]. The test conditions, given in Table 2, were based on those used by Foeth for the experimental Delft Foil test case [45]. The presented study considers only the centre section of the original twisted foil. This yields a 
Table 1: Mean force coefficients computed for a NACA00012 foil section at an angle of attack of $6^{\circ}$ for the purpose of numerical set-up validation.

\begin{tabular}{c|c|c} 
Method & $\overline{C l}$ & $\overline{C d}$ \\
\hline Experiment & 0.6533 & 0.00903 \\
RANS $k-\omega$ & 0.6412 & 0.01179 \\
LES & 0.6364 & 0.01096
\end{tabular}

geometry dominated by a single cavitation mechanism and allows a more in-depth analysis correlating the predicted flow features and noise signature. While relatively simple, the presented test case is subject to significant sheet cavitation, making it representative of some the key modelling challenges found on a marine propeller. Figure 3 shows the overview of the computational domain used.

Table 2: Summary of the dimensions of the foil and the flow properties used $[45,46]$.

\begin{tabular}{l|c|l} 
Parameter & Value & Unit \\
\hline Chord & 0.15 & $\mathrm{~m}$ \\
Angle of attack & 9 & $\mathrm{deg}$ \\
Span & 0.025 & $\mathrm{~m}$ \\
Inlet velocity & 6.97 & $\mathrm{~m} \mathrm{~s}^{-1}$ \\
Outlet pressure & 29 & $\mathrm{kPa}^{-}$ \\
Cavitation number, $\sigma$ & 1.07 & - \\
Water density, $\rho_{l}$ & 998 & $\mathrm{~kg} \mathrm{~m}^{-3}$ \\
Vapour density, $\rho_{v}$ & 0.023 & $\mathrm{~kg} \mathrm{~m}^{-3}$ \\
Water kinematic viscosity, $v_{l}$ & $10^{-6}$ & $\mathrm{~kg} \mathrm{~m}^{-2}$ \\
Vapour kinematic viscosity, $v_{v}$ & $4.273 \cdot 10^{-6}$ & $\mathrm{~kg} \mathrm{~m}^{-2}$ \\
Mean nucleation radius, $R_{0}$ & 50 & $\mu \mathrm{m}$ \\
Nuclei density, $n_{0}$ & $10^{8}$ & $\mathrm{~m}^{-3}$ \\
Saturated vapour pressure & 2970 & $\mathrm{~Pa}^{2}$
\end{tabular}

\subsection{Numerical setup}

A complete description of the boundary conditions used is given in Table 3. Wall functions were used in order to limit the cell count in the boundary layer region, following the approach outlined by Bensow et al. $[47,48]$. This offered a much smaller computational effort per unit of simulation time to be achieved, thus allowing for longer, more statistically relevant data records to be obtained. This type of wall treatment may reduce the accuracy of the simulation when resolving the re-entrant jets and other recirculating flow regions

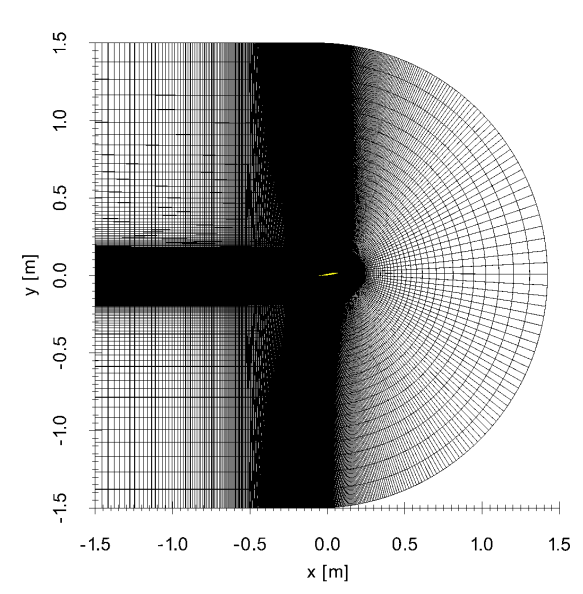

Figure 3: General arrangement of the C-grid used to discretise the domain.

close to the foil surface. On the other hand, a substantial amount of cavitation is located far away from the foil surface and so is less likely to be affected by the wall-function approach being adopted, making its use acceptable.

Temporal discretisation has been achieved by the use of a second-order implicit scheme. The maximum Courant number limit of 0.5 was needed to maintain stability of the LES simulation, yielding a fixed simulation time step of $2.5 \cdot 10^{-6} \mathrm{~s}$. The volume fraction was discretised using the van Leer scheme with interface compression in order to limit the interface smearing. A hybrid first-second order convection scheme was adopted in which $5 \%$ of upwinding was applied. The velocity and volume fraction fields were solved for using the preconditioned bi-conjugate gradient (PBiCG) solver with the diagonal incomplete lower upper (DILU) preconditioner whereas the generalised algebraic multigrid (GAMG) algorithm with the diagonal incomplete Cholesky (DIC) preconditioner was applied to the pressure equation.

Table 3: Boundary conditions set-up.

\begin{tabular}{c|c|c|c} 
Boundary & $U$ & $p$ & $\alpha$ \\
\hline Inlet & Dirichlet & Neumann & Dirichlet \\
Outlet & Neumann & Dirichlet & Neumann \\
Foil & No-slip & Fixed flux & Neumann \\
Top \& bottom & Slip & Neumann & Neumann \\
Span-normal & Slip & Neumann & Neumann
\end{tabular}




\subsection{Mesh}

A structured hexahedral mesh of c-grid topology, created using Pointwise, was used in order to divide the computational domain into a set of finite volumes. The grid, shown in Figures 3 and 4, consisted of 8.8 million cells overall, with most of them placed inside the boundary layer and the FW-H control surfaces. Small expansion ratios and fine cells were kept inside these mesh regions in order to limit the effect of dissipation and dispersion errors on the predicted noise. The foil was assumed to have a sharp trailing edge.

The computational grid consisted of 50 cells in the span-wise direction, aiming to provide $z^{+} \leq 150$ all over the foil surface. For the wall-normal spacing the mesh was designed to satisfy the $y^{+} \geq 30$ criterion required by the wall function used. An approximate $x^{+}$of 200 was also achieved on the foil surface. The domain was chosen to extend 10 chord lengths in all directions away from the foil in order to limit the effect of reflections on the flow field data on the FW-H control surface.

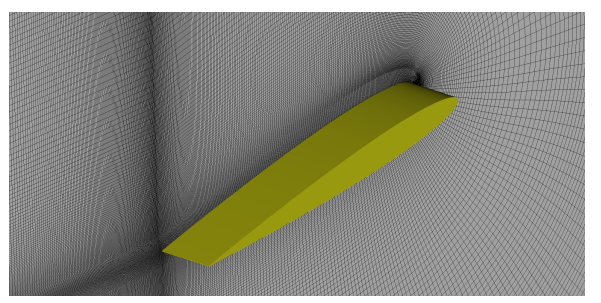

Figure 4: Detailed view of the mesh around the foil.

\subsection{Span-wise domain size}

The width of the numerical domain was chosen to be $0.025 \mathrm{~m}$, or $0.16 \mathrm{c}$. This could be considered sufficient for an aeroacoustic analysis, where it is often practical to simulate only a fraction of the span $[49,32,33,6]$. Doing so limits the size of the largest turbulent length scale that may be captured [32]. For a compressible simulation, the maximum permitted wavelength propagating in the span-wise direction is similarly limited, effectively only allowing for certain frequencies to be resolved in a three-dimensional manner for a given domain width [6].

In aeroacoustics a correction is usually applied to the numerical data in order to allow comparison with experimental results. A typical approach is outlined by Boudet et al. [32], where a coherence function is used to correct the radiated sound pressure levels given the simulation and experimental domain sizes.
To the authors' knowledge no such correction has been developed for cavitating hydrofoils. This implies that unless sufficient understanding of the threedimensional nature of the flow for a given cavitating hydrofoil is achieved and the characteristic span-wise lengths scales are identified no definite conclusions may be drawn with respect to the total sound pressure levels which could be encountered in reality. A relevant study was undertaken by Ji et al. [50], where cavitating flow around a hydrofoil was computed using LES with three different span-wise mesh resolutions. Authors pointed out that the vortical structures in the wake of the sheet cavity are particularly affected by refining the grid. This may be expected to affect the noise significantly as turbulence in the wake of the cavity sheet will act to make shedding unsteady and thus affecting the dominant source frequency. From a practical standpoint the computational cost of carrying out a sensitivity study by looking at several span-wise domain sizes and mesh densities was considered too great for this investigation and therefore only a limited section of the foil was used.

\subsection{Acoustic analysis}

Two FW-H control surfaces were used, as depicted in Figure 5. The smaller one was defined to end just outside of the maximum cavity extents observed in the initial simulations, aiming to prevent any local density variations. Its downstream plane was still in a fine mesh region in such a way as to enclose the convecting and collapsing cavities, as well as the most prominent wake structures.

A larger surface was also placed approximately twice as far away from the foil in a mesh region with a higher amount of cell stretching. This was intended to provide an estimate of how sensitive the acoustic solution is to the control surface location. Moreover, each of the control surfaces was also evaluated in an open-ended variant in order to study how much spurious noise the turbulent structures penetrating the downstream-most extents will generate. This phenomenon has been described, for instance, by Rahier et al. in the context of jet noise [51], and is caused by the noise sources leaving the control surface and not being accounted for using the volume integral which has been neglected.

Because slip boundaries were used in the span-wise direction it was appropriate to include the outside domain faces in the FW-H surface. By examining Equations (6) and (7) one may note, however, that these would not contribute significantly to the predicted noise levels because the angle between the radiation and normal directions would approach $\pi / 2$, rendering the 
pseudo-loading term contribution small, except for receivers far away from the domain centre-plane. As the normal velocity on a slip wall is also zero, the pseudothickness is not expected to contribute at all.

Figure 5 and Table 4 show the locations of the receivers which were placed around the foil, also highlighting the relative positions of the control surfaces employed. Six of the former were placed directly above the foil at mid-chord in order to evaluate the rate of decay of sound with distance from the foil. Two single listeners were located below and in front of the foil in order to help evaluate the directionality of the sound sources. Furthermore, an additional hydrophone was placed close to the foil downstream of the trailing edge in order to quantify the relative magnitudes of noise coming from the oscillating cavity and the turbulent wake. Two more receivers downstream on the centreline of the FW-H surface were used in order to shed more light on the directionality of the noise source, the relative importance of turbulence noise, and the effect of the FW-H control surface definition on the predicted acoustic signature. Finally, two receivers were situated $1 s$ and $2 s$ away from listener 5 in the span-wise direction in order to allow the effect of three-dimensionality of the noise source to be better understood.

Table 4: Locations of the receivers placed around the hydrofoil.

\begin{tabular}{c|c|c|c} 
Receiver & $\mathrm{x}[\mathrm{m}]$ & $\mathrm{y}[\mathrm{m}]$ & $\mathrm{z}[\mathrm{m}]$ \\
\hline 1 & 0.00 & 100.00 & 0.05 \\
2 & 0.00 & 75.00 & 0.05 \\
3 & 0.00 & 50.00 & 0.05 \\
4 & 0.00 & 25.00 & 0.05 \\
5 & 0.00 & 12.00 & 0.05 \\
6 & 0.00 & 3.00 & 0.05 \\
7 & 50.00 & 0.00 & 0.05 \\
8 & 0.00 & -50.00 & 0.05 \\
9 & -50.00 & 0.00 & 0.05 \\
10 & -0.23 & 3.00 & 0.05 \\
11 & -3.00 & 0.00 & 0.05 \\
12 & 0.00 & 12.00 & -0.10 \\
13 & 0.00 & 12.00 & -0.25
\end{tabular}

\subsection{Execution}

Prior to the discussed simulation the numerical grid had been subject to a convergence study whereby the relative change in the predicted steady-state, noncavitating force coefficients was investigated and found

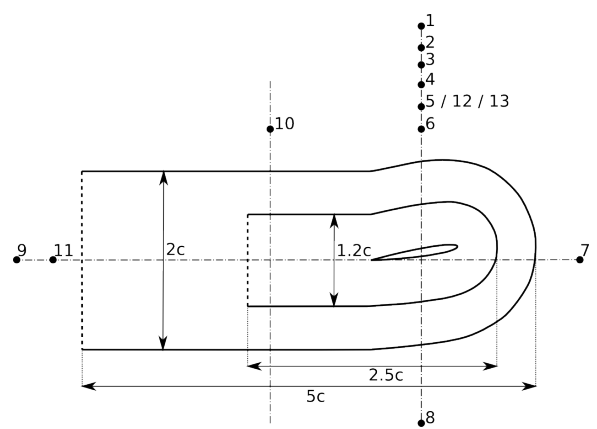

Figure 5: Positions of the hydrophones (black dots) used in the study relative to the foil and FW-H surfaces (solid lines), also indicating the downstream segments which were removed in the "open" configurations (thick dashed lines). Data surface dimensions given as a function of foil chord, $c$, distances of receives from the origin are not to scale.

to be $<2 \%$ when compared to a mesh with 25 million cells. The results of the steady-state computation were used to initialise the LES simulation, after which the unsteady solution was advanced in time for the period of $0.3 \mathrm{~s}$. During this time a ramp function was applied to the cavitation number in order to progressively introduce cavitation and avoid numerical instabilities at the beginning of the run [27]. This was done by modifying the saturated vapour pressure in (4) as a function of time, making it reach the desired value after $0.075 \mathrm{~s}$. After several initial cavitation cycles the transients had become negligible and the solution was sampled for $1 \mathrm{~s}$, or approximately 20 cavitation cycles, to provide data for the subsequent analysis.

Apart from the hydroacoustic analysis and global force coefficients, the total cavity volume was computed in the entire numerical domain using

$$
V=\sum_{i=0}^{N c e l l s} \frac{\min \left(0, \alpha_{\text {threshold }}-\alpha\right)}{\left|\alpha_{\text {threshold }}-\alpha\right|}(1.0-\alpha) \text { Vcell }_{i},
$$

where $\mathrm{Vcell}_{i}$ is the volume of a cell and $\alpha_{\text {threshold }}$ is a threshold value. Three different values were considered here: $0.1,0.5$, and 0.9 , where 1.0 corresponds to water with no vapour content.

The computation was carried out for a total of 360 CPU hours on the Iridis 4 supercomputer of the University of Southampton, excluding the initial run required to eliminate the transient flow features. It was run on 8 nodes with 16 dual $2.6 \mathrm{GHz}$ Intel Sandybridge processors each. The simulation required approximately 
$40 G B$ of RAM to execute, while the stand-alone acoustic analogy required the use of $20 G B$ and a negligible CPU-time when compared to the CFD.

\section{Results}

\subsection{Dominant flow features}

Figure 6 depicts an iso-contour of the volume fraction $\alpha=0.5$. Two large cavity structures are present above the mid-chord and downstream parts of the foil, with several smaller ones in between. A cavity sheet starting to develop around the leading edge of the foil may also be observed. Three-dimensionality of the flow is apparent from the cavity structures not being uniform in the span-wise direction across the foil.

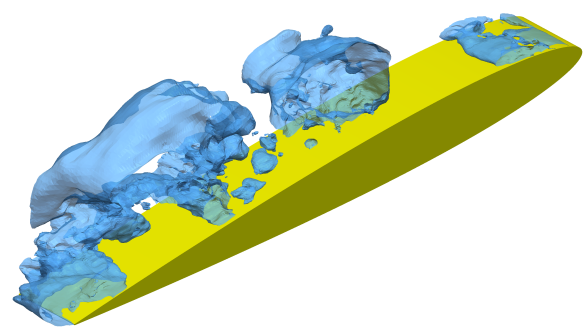

Figure 6: Sheet cavity growth and a set of large and smaller cavities present over the foil visualised using $\alpha=0.5$ iso-contour at source time $\tau=0.665 \mathrm{~s}$.

Figure 7 presents the distribution of the volume fraction at mid-span of the test section at four different simulation times. The presented flow data show a typical pattern whereby a sheet cavity grows from the leading edge of the foil up to a point where it becomes separated from the solid surface by a re-entrant jet of water. This splits the sheet into two or more cavity structures, with the upstream one remaining attached to the foil and the rest travelling downstream, after which the cycle repeats.

Figure 8 presents a distribution of the axial velocity over a mid-span cut plane, with a black iso-contour denoting the cavity interface defined as $\alpha=0.5$. A large cavity may be seen to be present just above the trailing edge of the foil and causing a severe velocity disturbance, in effect creating several small regions where the flow is slowed down or made to recirculate. Cavity structures from the previous cavitation cycles may be seen to have affected the wake of the hydrofoil by also having created velocity perturbations. Finally, a cavity sheet that had just been sheared off by a re-entrant jet may be seen around the leading edge. It is worth
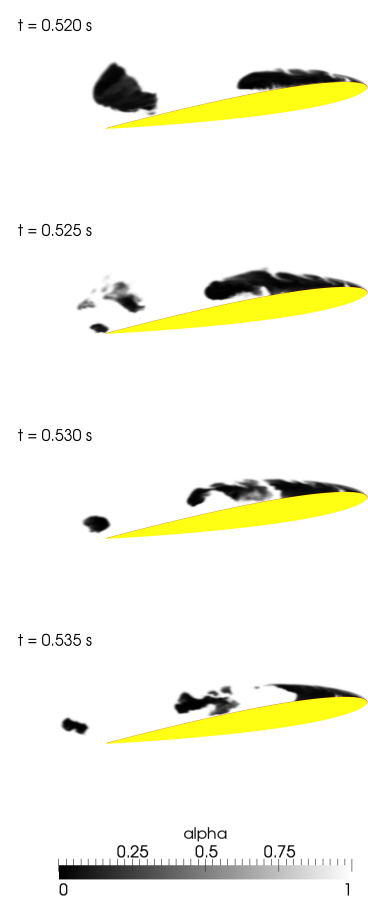

Figure 7: Four sequential snapshots of the cavity extent, visualised using the volume fraction distribution, at mid-span showing the passing of the re-entrant jet and shedding of cavity clouds. 
noting how the flow accelerates while circumnavigating this structure, indicating why a cavity sheet may sometimes be seen as an effective modification to the shape of the foil section.

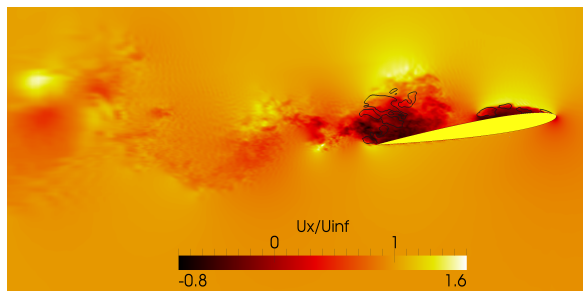

Figure 8: Span-normal cut showing stream-wise velocity distribution and the cavity interface $(\alpha=0.5)$.

These data indicate that the flow is dominated by the cavity sheet growth and shedding mechanisms, which affects the near- and far-field flow around the foil. It is thus apparent that understanding these relations is key to explaining the detail of the noise sources present and commenting on any inaccuracies of the modelling methods used.

\subsection{Effect of flow on the hydrofoil}

It is useful to examine the effect of the cavitation on the mean pressure coefficient distribution on the foil, as presented in Figure 9 for cavitating and non-cavitating conditions. The latter corresponds to the initial condition of the transient simulation computed using steadystate RANS method. Figure 10 shows a similar plot for the surface distribution of the mean volume fraction value on the upper half of the hydrofoil.

The two figures show that there exist five distinguishable regions on the upper surface of the foil. Around the low pressure peak, $x / c \leq 0.15$, where the foil is predominantly covered by sheet cavitation and experiences high fluctuations of the local volume fraction due to the periodic growth and destruction of the cavity sheet. Further downstream, for $x / c \in(0.15,0.45)$, cavitation is still largely attached to the foil surface. The positive gradient of the mean VOF value may be explained by the cavity sheet growing at an approximately steady rate, thus periodically advancing along the foil. The mean pressure coefficient sees a characteristic plateau from the leading edge up to this point. For the mid-span section of the original twisted foil this extended up to $x / c \approx 0.4$ [45], meaning that in the present simulation sheet cavitation extents approximately $5 \% c$ further downstream than in the case of the Delft Foil.
It is interesting to examine the time history of local volume fraction at $x / c=0.3$ shown in Figure 13. It shows two distinct time intervals during which the point on the foil surface is covered with vapour, corresponding to the presence of the sheet at this station. At times 0.725 and $0.775 s$ one may also note small perturbations in the signal but the fluid remains barely a mixture with a very low proportion of vapour, indicating that the local flow varies from one shedding cycle to another.

For $x / c \in(0.45,0.65)$ there appears to be an intermediate region with little cavitation touching the hydrofoil surface and the pressure coefficient starting to tend to the trailing edge value. Notable features are the step changes in the standard deviation and in the rate of change of the local volume fraction. From this point downstream fluctuations in the local pressure coefficient may be seen to steadily increase. Beyond this region the foil surface is almost entirely wetted with minimum fluctuations of the local volume fraction. Around the very trailing edge, $x / c \geq 0.9$, one may observe intermittent presence of cavities close to the foil surface.

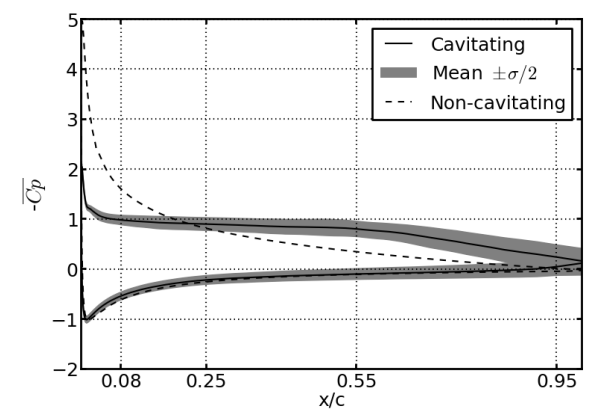

Figure 9: Time-averaged pressure coefficient distribution at mid-span of the foil surface, also showing the standard deviation, $\sigma$, at each location along the chord.

In order to allow a better explanation of the nature of the flow at the aforementioned stations along the foil power spectral densities of the local volume fraction and pressure coefficient are presented in Figures 12 and 11. The data focus on four locations approximately in the middle of each of the described regions, omitting the one between $x / c=0.65$ and 0.9 as it provides little additional information. The results show that everywhere except at $x / c=0.55$ the local quantities are dominated by frequency of $22 \mathrm{~Hz}$. At this particular station pressure coefficient may be seen to be broadband in nature and the local volume fraction experiences a peak around $30 \mathrm{~Hz}$, although its magnitude is comparatively small, suggesting the lack of a truly dominant component. The 


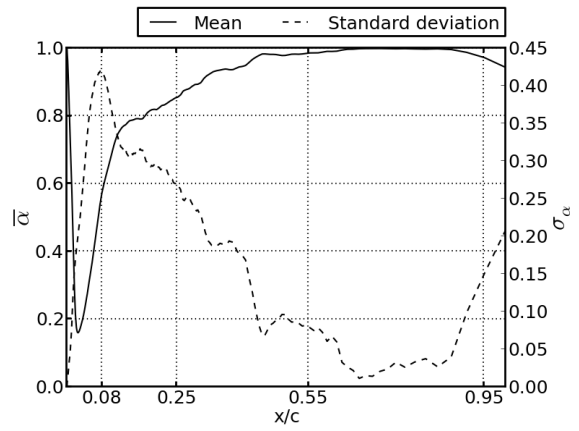

Figure 10: Distribution of the mean and standard deviation of the volume fraction on the upper surface of the foil, sampled at mid-span.

$20 \mathrm{~Hz}$ peak for the volume fraction near the trailing edge is also relatively broad, suggesting unsteadiness superimposed on the flow reliant on the shedding frequency.

Recalling the snapshots of the flow in Figures 8 and 7 , one may see how the locations on the foil where the sheet is present see very periodic behaviour but downstream of the cavity closure there exist more local flow instabilities. However, further downstream the flow is again dominated by regular passing of the shed clouds, explaining the periodicity of the signals around the trailing edge. The volume fraction trace there, however, gets affected by the shed clouds splitting into smaller elements and fluctuating in volume due to the action of vortical structures. This causes its signal to be more broadband than what is observed closer to the leading edge.

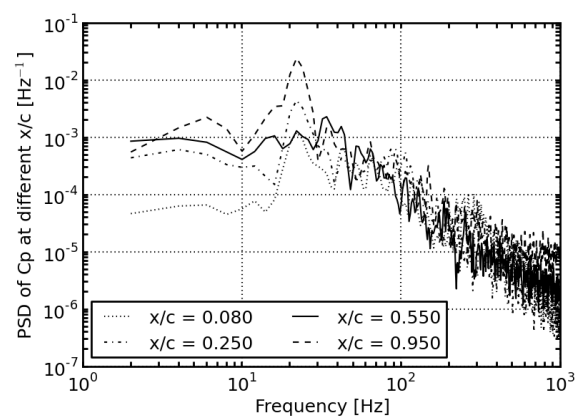

Figure 11: Power spectral density of the local pressure coefficient on the upper surface of the foil at $x / c=$ $0.08,0.25,0.55$, and 0.95 , sampled at mid-span.

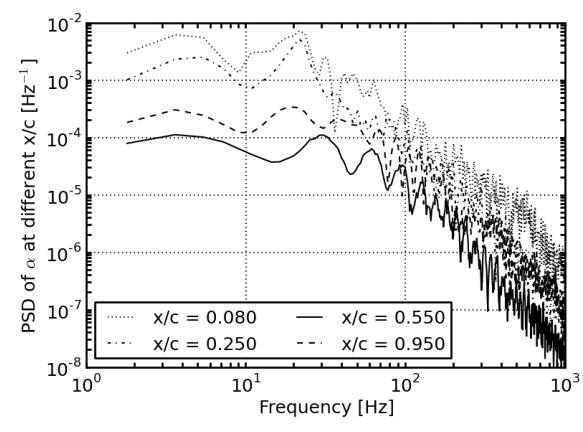

Figure 12: Power spectral density of the local volume fraction value on the upper surface of the foil at $x / c=$ $0.08,0.25,0.55$, and 0.95 , sampled at mid-span.

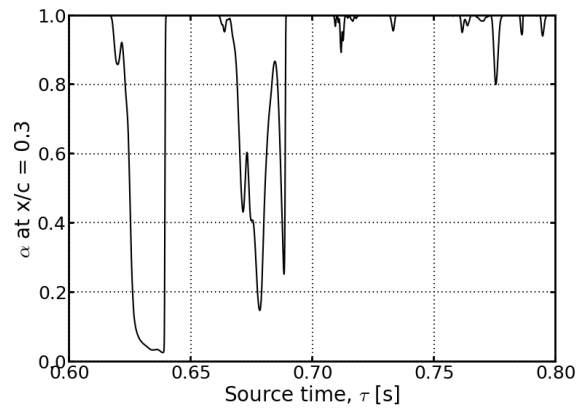

Figure 13: Time history of the local volume fraction value at $x / c=0.3$. 


\subsection{Integral foil loading}

Figure 14 presents part of the time history of the predicted lift coefficient. Strong fluctuations of the order of $\pm 20 \%$ about the non-cavitating, steady-state value of 0.914 may be seen in the signal. Nonetheless, the signal appears periodic and, as seen in its power spectral density function in Figure 15, it depends primarily on the shedding frequency. A notable feature are the sharp, narrow peaks preceding the maxima of the main $22 \mathrm{~Hz}$ waveform around times such as 0.675 and $0.775 \mathrm{~s}$.

Table 5 shows the convergence of the mean and standard deviation of the lift coefficient with extending the simulation duration. It may be seen that both of the quantities converge relatively quickly, suggesting that the simulation length has been sufficient in a statistical sense.

Table 5: Convergence of the mean and standard deviation of the lift coefficient with extending time window.

\begin{tabular}{c|c|c} 
Time window $[\mathrm{s}]$ & $\overline{C l}$ & $\sigma_{C l}$ \\
\hline $0.00-0.25$ & 0.8575 & 0.1553 \\
$0.00-0.50$ & 0.8761 & 0.1809 \\
$0.00-0.75$ & 0.8680 & 0.1805 \\
$0.00-1.00$ & 0.8667 & 0.1828
\end{tabular}

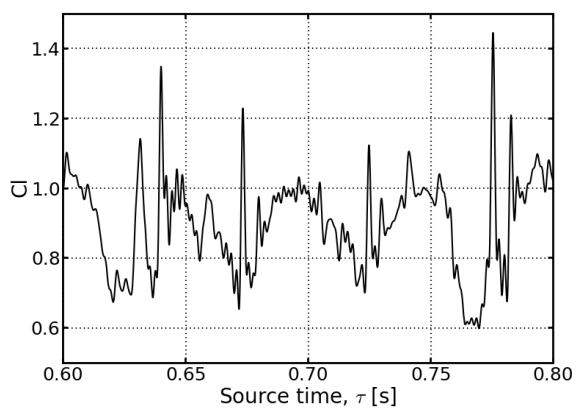

Figure 14: Lift coefficient time history for a time window corresponding to approximately 4 shedding cycles (treated using 5-element moving average and low-pass filtered at $500 \mathrm{~Hz}$ ).

\subsection{Cavity volume variation}

Figure 16 presents the time trace of the total cavity volume, computed using equation (15), for three different threshold values, namely $\alpha \leq 0.1, \alpha \leq 0.5$, and

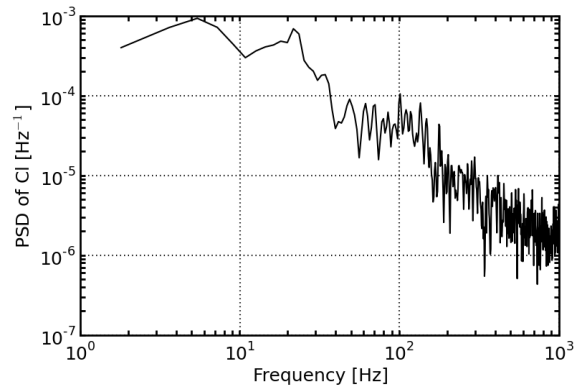

Figure 15: Power spectral density of the lift coefficient.

$\alpha \leq 0.9$. The signals may be seen to be periodic, although their magnitudes vary from cycle to cycle, particularly for the higher VOF threshold values. An important observation is that zero volume is reached only by the data series corresponding to the lowest threshold value. It should be noted that the data represents the total cavity volume present in the entire numerical domain and hence it is difficult to distinguish how much vapour is present in different types of cavitation structures without any additional analysis.

Figure 17 shows the difference between the cavity volume computed using the largest and smallest threshold values with reference to $\alpha \leq 0.5$ data. For the former the variation is insignificant (approximately 18\%). For the lowest VOF value, however, the predicted cavity volume is on average $65 \%$ smaller and sees higher fluctuations with standard deviation of $14 \%$ of the $\alpha \leq 0.5$ values, compared to $7 \%$ for $\alpha \leq 0.9$. Similar behaviour may be seen, for instance, in the study of a marine propeller by Gaggero et al. [52] or simulations of twisted hydrofoils by Ji et al. [53] and Li et al. [54].

It is important to look at the rate of change of the total cavity volume as it is expected to be directly responsible for driving noise generation. Figure 18 shows a portion of the data for the three volume fraction values used and the power spectral density of the signals is shown in Figure 19. The rate of change of volume may be seen to experience a peak around the frequency of $22 \mathrm{~Hz}$ for all VOF values, which has been identified as the main shedding frequency. This is significantly lower than the frequency reported for the original twisted foil which saw oscillations at $32 \mathrm{~Hz}$ [45]. In the case of the lowest VOF threshold there also exists a substantial amount of energy in the higher frequencies, which may indeed be seen by comparing the raw data in Figure 18.

Before proceeding with the acoustic analysis it is useful to identify particular stages of the cavitation cycle in order to better understand their effect on the flow, pay- 


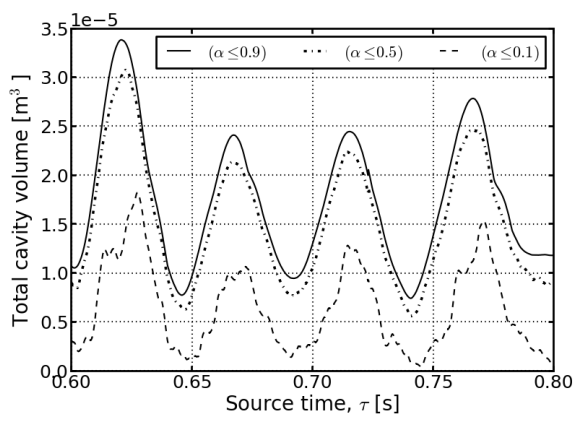

Figure 16: Total cavity volume time trace for various volume fraction thresholds.

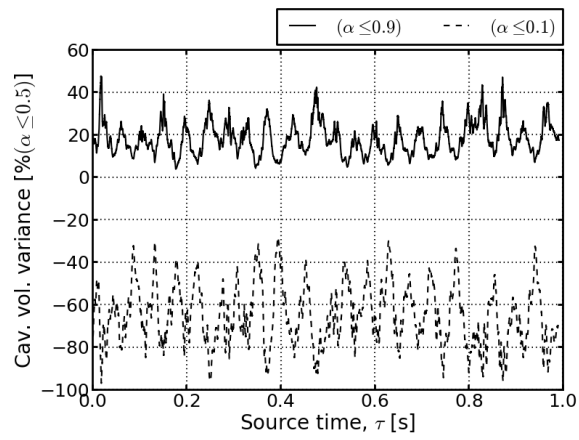

Figure 17: Difference between the total cavity volumes computed using the low $(\alpha \leq 0.1)$ and high $(\alpha \leq 0.9)$ volume fraction thresholds expressed as percentage of the volume for the mean interface value of $\alpha \leq 0.5$.

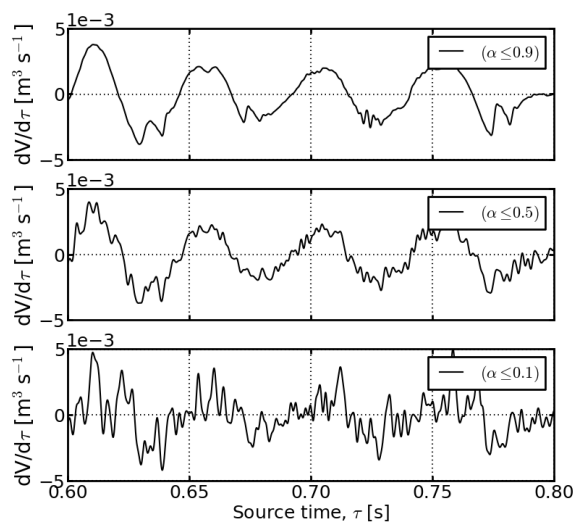

Figure 18: Source-time derivative of the total cavity volume time trace.

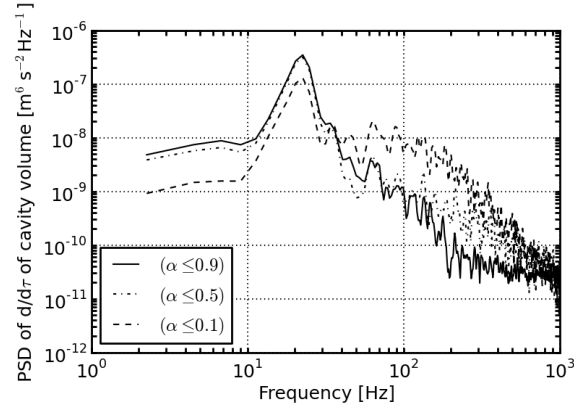

Figure 19: Power spectral density function of the time derivative of the total cavity volume for various volume fraction thresholds.

ing particular attention to Figures 7, 9, 10, 13, 16 and 18. First, the total cavity volume increases and reaches maximum rate of change at $\tau=0.61 \mathrm{~s}$, approximately a quarter of the way through the cycle of total volume oscillation. The growth of the volume continues and its maximum is reached at $\tau=0.62 \mathrm{~s}$. Shortly after this happens, the cavity sheet reaches the $30 \%$ of chord mark and, based on the mean profiles of surface volume fraction and pressure coefficient, one may deduce that the sheet will still grow beyond this point in time. Snapshots of the flow taken at this stage of the cavitation cycle indicate that around this time the volume of the cloud present in the wake starts decreasing which explains the reduction of the total amount of vapour in the numerical domain. At time $0.634 s$ minimum rate of change of the total volume is reached, which corresponds to an inflection point in the total cavity volume traces (more visible for $\tau=0.775 \mathrm{~s}$ ). This also corresponds to a small peak in the time derivative of the signals, right at the point where the primary waveform sees a trough. A closer inspection shows that around this time the total volume computed using the lowest VOF threshold sees a sharp peak. The re-entrant jet may be seen to pass over $x / c=0.3$ at $\tau=0.64 \mathrm{~s}$, leading to a subsequent decay of the total volume present in the domain, after which the shedding cycles continue.

Importantly, this pattern occurs for two of the cycles shown in the presented data, which are characterised by larger total volume, but may not be seen for the other two. This is because of the cavity sheet and the major cloud in the wake acting independently, although they follow approximately the same dominant frequency. Thus, if due to unsteadiness of the flow a phase shift is present time traces of their volumes do not fall on top of one another, causing a discontinuity in 
these signals.

This also highlights how the overall flow will be affected by the interactions of the two types of cavitation structures. This is evident in the $22 \mathrm{~Hz}$ frequency findings its way to time traces of nearly all of the considered flow quantities, such as surface pressure and integral forces. This is similar to the results presented by Ji et al. who found the surface pressure one the suction side of the foil has been observed to be affected predominantly by the cavity volume oscillation frequency [50].

\subsection{Effect of data surface choice of the FW-H solution}

Figure 20 shows the breakdown of the total sound pressure level (SPL) at receiver 3 into three terms, corresponding to the solutions of each of the integrals in Equation (7). Separate data series are plotted for each of the four control surface variants presented in Figure 5. It should be noted that for data shown in Figure 20 phase information has been lost and so adding the individual SPL spectra may not reproduce the actual total sound pressure level. SPL has been computed according to

$$
S P L=10 \log \left(\frac{p^{\prime}}{p_{\text {ref }}}\right)^{2},
$$

with the reference pressure level $p_{\text {ref }}$ of $1 \mu \mathrm{Pa}$. Time trace of the pseudo-thickness and pseudo-loading pressure terms for this receiver is also depicted in Figure 21.

The first striking observation in these data is that the noise signal is predominantly dependent on the pseudothickness term associated with mass flow through the porous control surface. This may be associated with the action of the oscillating cavities periodically pushing mass out of the region surrounding the foil. This is also evident as the spectral peak coincides with the $22 \mathrm{~Hz}$ maximum of the rate of change of total vapour volume signal. The pseudo-thickness term computed using all but the short-open control surface (CS) compares very well. For this particular CS the peak value is overestimated by approximately $5 d B$ and the associated waveform may be seen to suffer from the negative pressure peaks being of substantially higher amplitude. This may be explained by the fact that the smallest control surface ends close to the region where cavities exist and when its downstream end is left open a substantial part of the normal flow velocity remains unaccounted for.

However, closing the downstream surface may cause a different set of problems associated with the $p_{Q}^{\prime}$ volume integral of the FW-H equations being ignored. This causes the noise sources leaving the closed control surface, such as turbulent eddies, not being accounted for well enough. This may be seen in the presented data as an increase in the pseudo-loading (proportional and derivative) terms of the acoustic analogy caused by strong peaks appearing in the time signals of these terms, as seen in Figure 21. A noteworthy fact is that despite the large control surface being placed in a coarser mesh region the vortices still have a comparable effect on the predicted spurious noise as in the case of the small surface.

Another important obstacle in using an open-ended control surface is that, by virtue of the pseudo-loading terms being dependent on the dot product of radiation and normal directions, its faces will contribute more to the downstream receivers. To evaluate the importance of this the SPL of individual FW-H terms was plotted for receiver 6 in Figure 22. One may note that, as expected, the relative importance of the pseudo-loading terms becomes higher and the result obtained using the short-closed control surface diverges the most of the remaining predictions. The latter indicates that the spurious noise source is indeed the strongest where the mesh is finest and the vortices are best preserved. This may be visualised by examining data in Figure 23 showing how the turbulence in the flow interacts with the control surface inducing localised noise sources. The presented noise data depicts the instantaneous FW-H contribution of each of the faces constituting the data surface. This has been divided by the face area of each face of the control surface, thus yielding a quantity which may be interpreted as an intensity. This allows a more accurate comparison of faces with different areas. It is also interesting to note the high turbulence levels around the foil where cavitation is present.

Following this investigation it has been decided to use the open variant of the large control surface in the subsequent acoustic analysis as it appears to be able to capture the dominant pseudo-thickness FW-H term and is not affected by the spurious noise sources in the wake.

\subsection{Nature of the sound source}

Figure 24 shows time history of the acoustic pressure at receiver 1 plotted as a function of the source time by applying a phase shift of $|r|_{\text {median }} / c_{0}$ where $|r|_{\text {median }}$ is the median source-receiver distance of the control surface faces. Viewing the data in this way allows the sound history to be compared directly with the time derivative of the total cavity volume in Figure 18.

Points on the $d V / d \tau$ curves with steepest slope may be seen to coincide with the peaks and troughs in the pressure waveforms, showing a direct relationship between the two terms. Moreover, the noise signal has a 


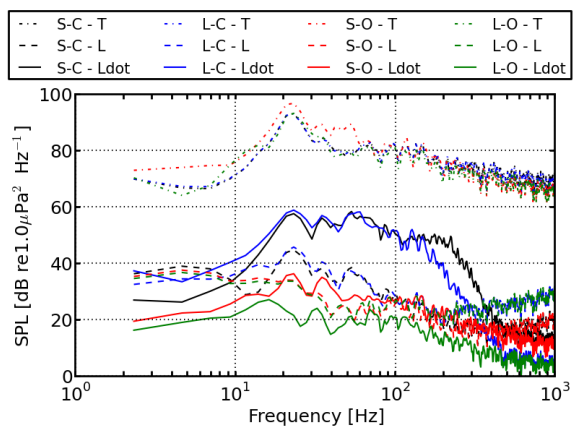

Figure 20: Sound pressure level predicted at receiver 3 showing the contributions of different FW-H components, corresponding to individual terms in equations (7). Data for short (S) and large (L) control surfaces in closed $(\mathrm{C})$ and open-ended $(\mathrm{O})$ variants.

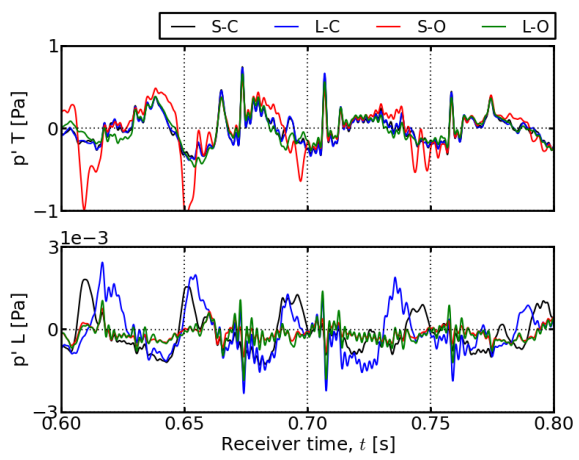

Figure 21: Time trace of the pseudo-thickness (T) and pseudo-loading (L) FW-H terms for receiver 3 for the four control surface variants used.

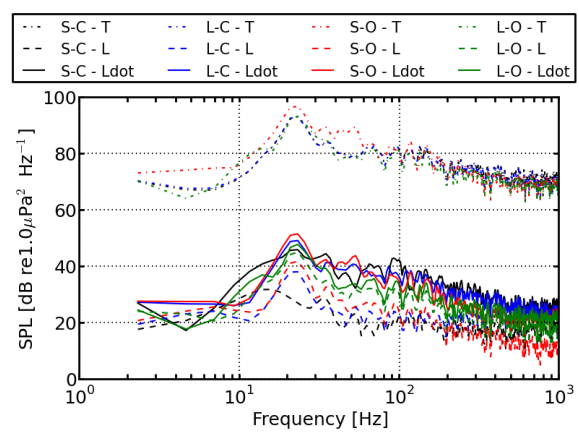

Figure 22: Sound pressure level predicted at receiver 9 showing the contributions of different FW-H components, corresponding to individual terms in equations (7), for the control surfaces used.

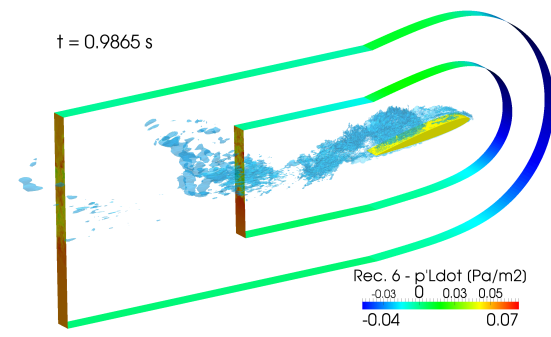

Figure 23: Distribution of the derivative part of the pseudo-loading FW-H term in Eq. (7b) for receiver 9 on both control surfaces used, also showing a vorticity iso-contour $\omega=1000 \mathrm{~s}^{-1}$.

certain amount of higher frequency components. These could be due to the pseudo-loading, but also the pseudothickness term, as seen in Figure 21. This shows that, while the dominant frequency of the signal seems to be excited by the entire vapour region up to high threshold VOF values, the perturbations in the more coherent cavity regions may also play an important role in deciding the final pressure signal.

An important observation is the presence of strong, impulse-like peaks in the pressure signals. These precede the occurrence of the maxima of the dominant $22 \mathrm{~Hz}$ waveform, much in the same fashion as has been seen for the lift coefficient in Figure 14. These may be seen to coincide in time with the troughs of the time derivative of the total cavity volume curves, which have previously been identified to be associated with desinence of the cloud cavity present downstream. Notably, the noise peaks are most prominent during the cavitation cycles when largest cloud cavity, corresponding to largest total vapour volume, is observed. Figure 25 shows two consecutive snapshots of the flow which indicate how a cloud around the trailing edge of the foil rapidly loses a significant part of its volume. The presented pressure contours indicate that a rapid increase in surface pressure on the foil is observed. It is this mechanism that is responsible for generation of the discussed peaks in the integral forces and radiated pressure. A key thing to notice is that the magnitude of the narrow radiated pressure peaks is at least as large as that of the low-frequency oscillations.

\subsection{Sound field characterisation}

It is worth examining how the sound field radiated from the hydrofoil in order to better understand its directivity and the relative importance of particular flow regions in terms of noise generation. Figure 26 shows 


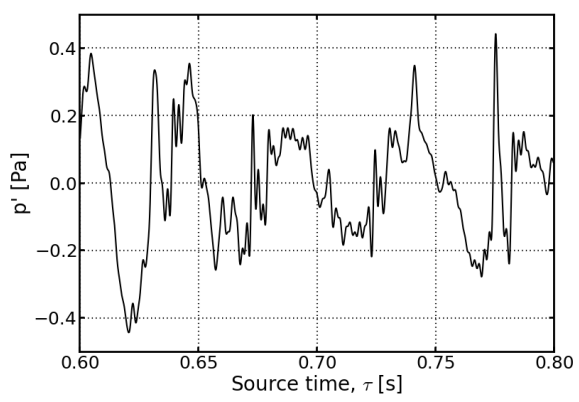

Figure 24: Time trace of the predicted acoustic pressure at receiver 3 shown as a function of the source time (corrected using median source-receiver distance).
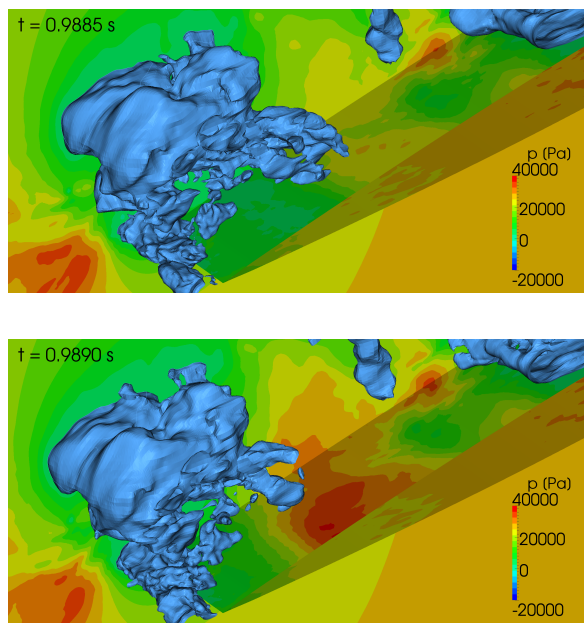

Figure 25: Consecutive snapshots of the cavity interface $(\alpha=0.5)$ and pressure on the surface of the foil and a span-wise extent of the domain before and after a violent collapse of a part of the cavity takes place. Cavity cloud is located above the trailing edge, downstream end of the growing sheet also seen in the top-right corner. time series data for receivers displaced by 1 and 2 chord lengths in the span-wise direction from receiver 3 . One may note very little difference between the waveforms. This may be explained by the radiated sound being dominated by the pseudo-thickness term contribution of the FW-H equations (see Eq. (7)), which depends on the magnitude of the radiation vector, $r$, and on the control surface normal direction, $\mathbf{n}$, as seen in Figure 21, for instance.

Figure 27 presents time signals of the predicted radiated pressure for receivers $3 \mathrm{~m}$ away from the foil just above its mid-chord, above the wake, and directly downstream. The purpose of analysing these data was to examine whether with reducing the source-receiver distance the pseudo-loading FW-H terms will increase in magnitude sufficiently to start playing a significant role in the resultant total pressure waveform shape. As may be seen this was not the case since the predicted signals resemble the ones at other receivers closely, apart from their magnitude being significantly higher, as expected. It is also worth noting how the same behaviour is observed for receivers below and in front of the hydrofoil, as shown in Figure 28.

It is worth recalling that for a porous FW-H formulation the individual terms lose their physical meaning and so the pseudo-thickness term may not be directly associated with a monopole source being present in the fluid. However, all of the result presented above indicate that the dominant noise source is indeed of monopole nature. As a final confirmation of this, Figures 29 and 30 present the radial variation of the sound pressure level emitted at the dominant $22 \mathrm{~Hz}$ frequency at receivers directly above the hydrofoil. It is also useful to note that at intermediate frequencies the spectra see a falloff slightly slower than $f^{-2}$, which stands in general agreement with a range of cavitation noise experiments presented in the literature [55].

In the present simulation the cavities are not spherical, but one may attempt to approximate the sound they radiate by finding their equivalent, idealised radius, and computing the pressure a spherical bubble of that size would produce in a quiescent liquid. From the time trace of the total cavity volume, using $\alpha \leq 0.5$, the average maximum volume of the bubble during each cavitation cycle was found to be $V_{\max } \approx 2.0 \cdot 10^{-5} \mathrm{~m}^{-3}$. Assuming spherical shape, this yields $R_{\max } \approx 0.017 \mathrm{~m}$. The bubble may now be assumed to undergo simple harmonic motion with its radius oscillating between $R_{\text {max }}$ and zero at $22 \mathrm{~Hz}$. The pressure radiated by a spherical bubble may be described using Equation (9), where the source strength, $Q$, is the second time derivative of volume change of the bubble, and may be written for a 
spherical cavity of radius $R$ as

$$
Q=\ddot{V}=4 \pi\left(R^{2} \ddot{R}+2 R \dot{R}\right) .
$$

This yields radiated sound at a point [56]

$$
p(\mathbf{x}, t)=\left.\frac{\rho}{|\mathbf{x}-\mathbf{y}|}\left(R \ddot{R}+2 R \dot{R}^{2}\right)\right|_{\tau} .
$$

Naturally, both $R$ and its source time derivatives in Equations (17) and (18) will be governed by the Rayleigh-Plesset equation [8], thus making determination of radiated pressure at a point more complex in reality $[57,56]$.

The equivalent radius derived from Figure 16 has been found to cause pressure oscillations of a much greater amplitude than was observed in the numerical data. By an empirical fit the predicted noise data has been found to correspond to a bubble of radius with maximum radius of approximately $15 \% R_{\max }$. This data is also plotted in Figure 30 for comparison.

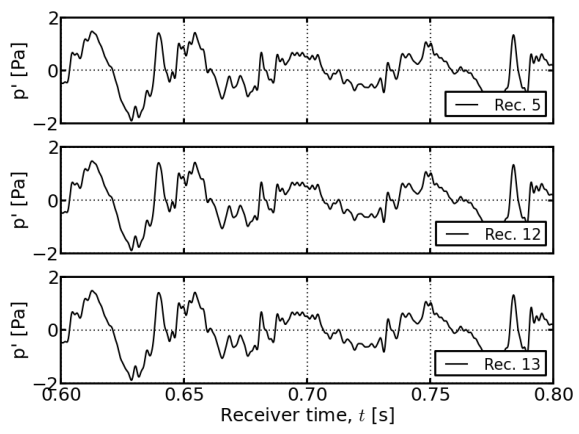

Figure 26: The effect of moving receiver 5 by 1 and 2 chord lengths in the span-wise direction to positions of receivers 12 and 13 , respectively.

\section{Discussion}

No experimental data for the particular case study considered here exist. However, two relevant data sets for the same foil profile have been presented in the literature. The first is the Delft twisted foil experiments by Foeth [45] on whose setup the present simulation has been designed. Secondly, a series of experiments on the NACA0009 profile with a blunt trailing edge has been conducted by Dupont [58] and Ait Bouziad [59]. The latter did look at more intermediate angles of attack $\left(5^{\circ}\right)$ and higher Reynolds numbers $\left(R e \approx 2 \cdot 10^{6}\right)$. This allows for qualitative comparison of the flow regimes to still be carried out.

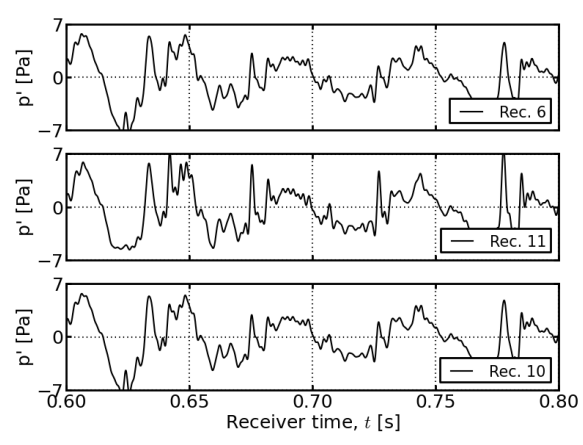

Figure 27: Acoustic pressure predicted at receivers downstream and above the wake, compared with the baseline receiver above mid-chord of the foil. Listeners located $12 m$ from the origin.

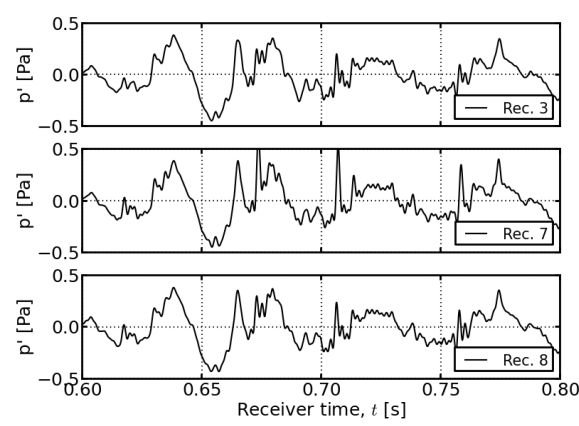

Figure 28: Pressure oscillations computed for far-field receivers in front and below the foil.

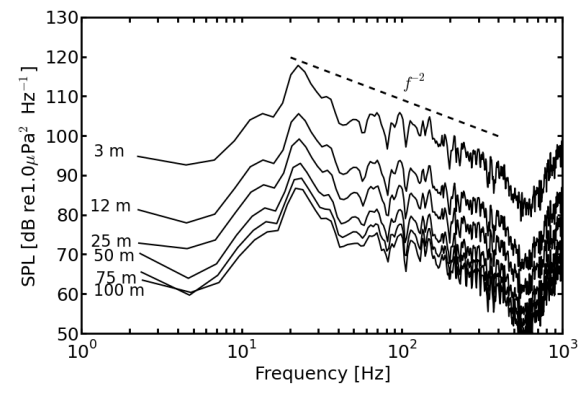

Figure 29: Variation of the predicted sound pressure level for receivers directly above the foil (1 to 6) with source-receiver distance. 


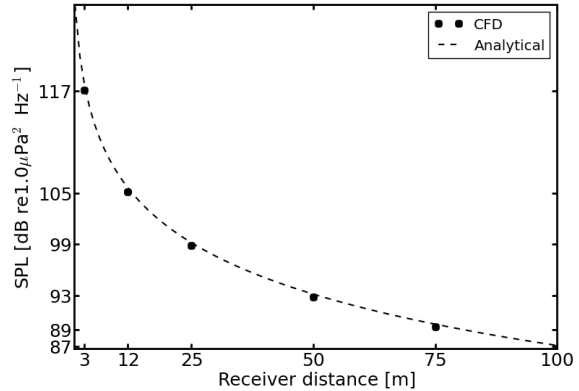

Figure 30: Decay of the sound pressure level predicted at the dominant frequency of $22 \mathrm{~Hz}$ with source receiver distance for receivers 1 to 6 . Data for an idealised spherical bubble acting as a monopole noise source in accordance with Equation (18) plotted for comparison.

Relatively large difference in the shedding mechanism has been observed between the considered hydrofoil and the twisted Delft Foil. In this case a constant angle of attack section which corresponds to the profile used at mid-span of the twisted foil was used. Therefore, the shedding mechanism predicted here has been generally two-dimensional. In the experimental study highly three-dimensional behaviour has been reported, with the cavity sheet collapse being affected by re-entrant jets in the stream-wise and span-wise direction. Because of this the cavity sheet in the present case is likely to reach further downstream and experience shedding at a lower frequency. In the present study the Strouhal number defined based on the maximum sheet cavity length (taken as $0.6 c$ based on results in Figure 10) and free-stream velocity has been computed to be 0.284, while the experimental data by Ait Bouziad for cavitation number 1.2 quoted it to be 0.36 . It thus seems possible that the shedding frequency is under-predicted in the current simulation, which would have most likely been caused by the subgrid model introducing too much viscosity in the region of cavity closure $[16,60]$. Such behaviour has been previously discussed in the literature, for instance in the study by Wikstrom in relation to a one-equation subgrid sale model [16]. Since the comparison made remains only qualitative it is impossible to comment on the exact magnitude of errors. At the same time, the observed pressure coefficient and cavity extents agree well with both experimental studies referred to. Hence the key discussion made here about the interactions between the flow and the acoustic analogy remain valid.

One of the key observations has been the tendency for the $2 \mathrm{D}$ cyclic cavity sheets to turn into more three- dimensional vapour structures in the wake of the foil. This caused the flow downstream to show less spanwise correlation than it did around the leading edge, explaining the differences seen in total cavity volumes predicted between consecutive cycles. Because of this unsteadiness, a significant amount broadband pressure fluctuations on the foil has been observed. Referring to the data presented by Bretschneider et al. for a NACA0015 section, one may see that for an untwisted foil experiencing sheet cavitation severe amount of variation along the span may be seen at any one time. This reportedly also manifests itself in a severely fluctuating pressure signal for a transducer in direct vicinity of the foil, likely having a similar effect on the foil loading [61].

Furthermore, the predicted cavities have been seen not to always be well defined, which is evident from the volume fraction adopting intermediate values between 0 and 1 in large volumes of the computational domain, particularly downstream of the sheet cavity. Because of this, choosing volume fraction thresholds different than the commonly accepted interface value of $\alpha=0.5$ leads to very different flow properties being observed and computed. This emphasises the importance of conducting of a sensitivity study similar to the one presented here when considering transient multiphase flow problems.

These regions of intermediate vapour fraction values may be physically interpreted as a mixture of smaller bubbles being carried by the fluid. Such cavities are known to have a high noise-generating potential as they oscillate subject to a varying pressure field $[62,63,64,65]$. However, due to the way they are described in the volume-of-fluid approach this behaviour may not be captured.

Another limitation of the current methodology stems from the incompressible flow assumption and the nature of the mass transfer cavitation model. It has been shown that rapid desinence of cloud cavities may have a significant effect on the local pressure on the foil, the integral force coefficients, but also on the radiated noise. However, such collapse mechanism is significantly affected by flow compressibility and requires the bubble mechanics to be resolved to very small spatial and temporal scales [66]. It thus follows that because the adopted theoretical model cannot predict shock waves associated with cavity collapse the impulse-like character of the associated radiated pressure may not be predicted accurately. This behaviour is further enhanced by the fact that while the numerical grid in this study may be considered fine when compared to those typically used in marine applications, it would be far too 
coarse to capture the final stages of the collapse, even if compressibility had been accounted for. One may thus argue that, while the noise due to large-scale cavity interface velocities may be predicted reasonably well using the presented method, the peak noise values associated with the final collapse events are likely to be predicted incorrectly due to insufficient mesh density and an incompressible flow solution being employed.

It has been shown that even for receivers in a relative proximity to the hydrofoil the dominant part of the noise is caused by the large-scale fluctuations of the cavities. This kind of excitation has been found to manifest itself through the pseudo-thickness term of the FW-H acoustic analogy, which is independent of the radiation vector and only on the radiation distance. Making use of this property, it has been demonstrated that ignoring the volume integral and leaving the downstream end of the FW-H control surface open is a viable means of reducing the complexity of the simulations without introducing significant errors, even for downstream receivers.

However, if the amount of cavitation present would be smaller, such as could be expected on a welldesigned ship propeller close to its operating point, one could expect the relative importance of the turbulent noise from the wake to become increasingly important. This would hold particularly true in the case of a propeller operating in a non-uniform, unsteady inflow. In such a case neglecting the volume integral and using an open-ended control surface could lead to significant errors, especially when considering radiation directions aligned with the flow. Such a situation has been described, for instance, by Ianniello et al. who considered the noise originating from vortices in the wake of a non-cavitating marine propeller [67]. This stresses the importance of searching for viable alternatives to using open FW-H control surfaces, such as the ones discussed by Rahier et al. [51] or Sinayoko et al. [68]. An intermediate option would be to solve the volume integral in the wake region only and neglecting it further away where quadrupole sources may be expected to be negligible.

The dominant noise source has also been demonstrated to be of monopole nature, as expected from the literature for oscillating vapour volumes. Because of this property and the way the Ffowcs Williams - Hawkings acoustic analogy is formulated, the noise field showed little variation in the span-wise direction, even if the radiation vector joining the surface of the foil and the virtual hydrophone does not penetrate the control surface. This could potentially mean that results obtained from a simulation similar to the one presented here could be used without much need for applying span corrections, like the ones discussed for turbulence noise over wings $[49,32,33]$. One has to be careful, however, as it has been shown in this work and in the literature that sheet cavitation is likely to develop threedimensional features, even if the analogous wetted flow may well be two-dimensional [50]. At this stage it is therefore unclear how changing the span of the hydrofoil section would affect the radiated noise.

\section{Conclusions}

Despite several areas requiring further work, the present methodology, involving Large Eddy Simulation, a mass transfer cavitation model, and a porous Ffowcs Williams-Hawkings acoustic analogy, has been demonstrated to capture the dominant noise source associated with the cavity volume oscillations well. A direct link between the radiated noise and elements of the waveform of the time derivative of the total vapour volume has been indicated. In accordance with what had been expected from the literature, the cavitationinduced, low-frequency noise has been demonstrated to be monopole in nature. This is particularly important given that the acoustic method did not use any modelling assumptions in this context.

Noise due to the oscillating cavities has also been found to contribute overwhelmingly to the pseudothickness term of the acoustic analogy, even for receivers closer than one wavelength away from the hydrofoil. This allowed for the volume integral of the Ffowcs Williams-Hawkings acoustic analogy to be ignored and the downstream end of the porous integration surface to be left open, removing the problem of spurious noise originating from turbulent sound sources leaving the domain, without the predictions being affected significantly. This has been recognised only as a temporary solution as on marine propellers operating in a wake relative importance of non-linear noise sources in the wake may increase.

Moreover, because the dominant FW-H term is independent of the radiation direction the predicted radiated pressure results vary little in the span-wise direction. This appears to allow a comparatively small span-wise extent of the numerical domain to be chosen, provided that is large enough to capture three-dimensionality of the cavitation structures. Further studies in this respect are needed, however, for definitive conclusions to be drawn.

A noticeable limitation of the presented methodology is its inability to accurately resolve the flow when the cavities reduce to the same order as the grid size, 
particularly during rapid collapse phases. Further inaccuracies in capturing this process stem from the use of an incompressible flow formulation, common in marine applications. Both of these factors have a negative effect on the accuracy of the predicted noise, particularly in the high-frequency region.

This part of the sound pressure level spectrum is also lacking the contribution of the volume fluctuations of small bubbles as these are omitted in the simulation. Given that the broadband cavitation noise has been identified as one of the main environmental concerns, these issues may be seen as some of the most pressing challenges in terms of modelling development required for robust hydroacoustic predictions for ships using Computational Fluid Dynamics [4]. It would therefore be beneficial to explore alternative cavitation modelling approaches, such as hybrid VOF-particle tracking or inhomogeneous flow models [69], in order to improve the current model in this respect. One could also consider using automatic grid refinement to resolve the interface, as well as smaller bubbles and their collapse, better. However, all of these methods would likely be associated with an increased computational effort [24, 70, 71].

Finally, it has also been indicated that a large variety of data may be used to support discussion and gain insight into the nature of the cavitating flows. However, it could be seen that a large fraction of this information is integral in nature and does not necessarily provide the means to study individual phenomena in detail. This applies in particular to topologically distinguishing between cavity sheets and cloud volumes, tracking the progress of and energy contained within re-entrant jets, and accurately localising individual noise sources in four-dimensional space. A further development of the post-processing methods is therefore needed to deepen the understanding of cavitating flows and support experimental studies.

\section{Acknowledgements}

The authors would like to acknowledge the use of OpenFOAM 2.2.2 C++ libraries and the Iridis 4 supercomputer of the University of Southampton for all of the presented simulations. We would also like to thank the reviewers for their input which significantly improved the quality of this work.

[1] P. Kellet, O. Turan, A. Incecik, Underwater Noise and Marine Wildlife: Current and Future Implication and Assessment, in: A. Yücel Odaba $\AA$ Colloquium Series, 6th-7th November, Istanbul, Turkey, 2014, pp. 63-69.

[2] EU FP7, SONIC Project (2014)

URL http: //www. sonic-project.eu/
[3] EU FP7, AQUO Project (2014) URL http: //www . aquo . eu/

[4] H. Bertschneider, J. Bosschers, G. H. Choi, E. Ciappi, T. Farabee, C. Kawakita, D. Tang, Specialist Committee on Hydrodynamic Noise, Tech. rep., ITTC (2014).

[5] T. Brooks, Airfoil tip vortex formation noise, in: American Institute of Aeronautics and Astronautics and NASA, Aeroacoustics Conference, Williamsburg, VA, 1984.

[6] T. P. Lloyd, Large eddy simulations of inflow turbulence noise: application to tidal turbines, Ph.D. thesis, University of Southampton (2013).

[7] S. Ianniello, R. Muscari, A. D. Mascio, Ship underwater noise assessment by the acoustic analogy, part III: measurements versus numerical predictions on a full-scale ship, Journal of Marine Science and Technologydoi:10.1007/s00773-013-0228-z.

[8] M. S. Plesset, A. Prosperetti, Bubble dynamics and cavitation, Annual Review of Fluid Mechanics 9 (1) (1977) 145-185.

[9] R. E. Bensow, G. Bark, N. Lu, Hydrodynamic Mechanisms in Cavitation Erosion, in: International Symposium on Cavitation, no. 148 , Singapore, 2012 , pp. 1-6.

[10] H. Kanfoudi, H. Lamloumi, R. Zgolli, Numerical Investigation for Steady and Unsteady Cavitating Flows, Advances in Modeling of Fluid Dynamics (2012) 87-96.

[11] H. Seol, J.-C. Suh, S. Lee, Development of hybrid method for the prediction of underwater propeller noise, Journal of Sound and Vibration 288 (1-2) (2005) 345-360. doi:10.1016/j.jsv.2005.01.015.

[12] I. Kirsteins, P. Clark, L. Atlas, Maximum-likelihood estimation of propeller noise modulation characteristics, in: Proc. Underwater Acoustic Measurements, 2011.

[13] G. Wang, M. Ostoja-Starzewski, Large eddy simulation of a sheet/cloud cavitation on a NACA0015 hydrofoil, Applied Mathematical Modelling 31 (3) (2007) 417-447. doi:10.1016/j.apm.2005.11.019.

[14] J. H. Seo, Y. J. Moon, B. R. Shin, Prediction of cavitating flow noise by direct numerical simulation, Journal of Computational Physics 227 (13) (2008) 6511-6531. doi:10.1016/j.jcp.2008.03.016.

[15] J. Decaix, E. Goncalvès, Compressible effects modeling in turbulent cavitating flows, European Journal of Mechanics - B/Fluids 39 (2013) 11-31. doi:10.1016/j.euromechflu.2012.12.001.

[16] M. Wikstrom, Approaching Large eddy simulation of cavitating flows for marine applications, Ph.D. thesis, Chalmers University of Technology (2006).

[17] X. Chen, N. D. Sandham, X. Zhang, Cavity flow noise predictions, Tech. Rep. February, Aerodynamics \& Flight MEchanics Group, University of Southampton (2007).

[18] J. Ffowcs Williams, D. L. Hawkings, Sound generation by turbulence and surfaces in arbitrary motion, Philosophical Transactions of the Royal Society of London Series A, Mathematical and Physical Sciences 264 (1151) (1969) 321-342.

[19] P. Di Francescantonio, A new boundary integral formulation for the prediction of sound radiation, Journal of Sound and Vibration 202 (4) (1997) 491-509. doi:10.1006/jsvi.1996.0843.

[20] J. Sauer, G. H. Schnerr, Development of a new cavitation model based on bubble dynamics, Zeitschrift für Angewandte Mathematik und Mechanik 81 (2001) 561-562.

[21] F. Salvatore, S. Ianniello, Preliminary results on acoustic modelling of cavitating propellers, in: IABEM 2002, International Association for Boundary Element Methods, UT Austin, TX, USA, 2002.

[22] K. Park, H. Seol, W. Choi, S. Lee, Numerical prediction of tip vortex cavitation behavior and noise considering nuclei size and distribution, Applied Acoustics 70 (5) (2009) 674-680. 
doi:10.1016/j.apacoust.2008.08.003.

[23] C. T. Hsiao, A. Jain, G. L. Chahine, Effect of gas diffusion on bubble entrainment and dynamics around a propeller, in: 26th Symposium on Naval Hydrodynamics, no. September, Rome, Italy, 2006, pp. 17-22.

[24] A. Vallier, J. Revstedt, H. Nilsson, A multi-scale approach for modelling cavitation on hydrofoils, implemented in OpenFOAM, in: 8th International OpenFOAM Workshop, Jeju, Korea, 2013.

[25] T. G. Leighton, a. J. Walton, An experimental study of the sound emitted from gas bubbles in a liquid, European Journal of Physics 8 (2) (1987) 98-104. doi:10.1088/0143-0807/8/2/005.

[26] C. Brennen, Cavitation Bubble Dynamics and Noise Production, in: 6th International Workshop on Multiphase Flow, 1993, pp. 3-28.

[27] R. Bensow, Simulation of the unsteady cavitation on the the Delft Twist11 foil using RANS, DES and LES, in: 2nd International Symposium on Marine Propulsors, no. June, Hamburg, Germany, 2011.

[28] A. Vallier, Simulations of cavitation-from the large vapour structures to the small bubble dynamics, Ph.D. thesis, Lund University (2013).

[29] A. H. Koop, Numerical simulation of unsteady threedimensional sheet cavitation, Ph.D. thesis, University of Twente, Enschede, The Netherlands (sep 2008). doi:10.3990/1.9789036527019.

[30] N. Lu, U. Svennberg, G. Bark, R. E. Bensow, Numerical simulations of the cavitating flow on a marine propeller, in: Eighth International Symposium on Cavitation CAV 2012, no. Cav, 2012, pp. 978-981. doi:10.3850/978-981-07-2826-7.

[31] K. S. Brentner, F. Farassat, An analytical comparison of the acoustic analogy and Kirchhoff formulation for moving surfaces, in: American Helicopter Society 53rd Annual Forum, Vol. 36, Virginia Beach, Virginia, USA, April 29-May 1, 1997 , pp. $1-17$.

[32] J. Boudet, N. Grosjean, M. C. Jacob, Wake-airfoil interaction as broadband noise source: a large-eddy simulation study, Aeroacoustics 4 (1) (2005) 93 - 115. doi:10.1260/1475472053730093.

[33] J. C. Giret, A. Sengissen, S. Moreau, M. Sanjosé, J. C. Jouhaud, Prediction of the sound generated by a rod-airfoil configuration using a compressible unstructured LES solver and a FW-H analogy, 18th AIAA/CEAS Aeroacoustics Conference (33rd AIAA Aeroacoustics Conference) (2012) 118doi: $10.2514 / 6.2012-2058$.

[34] Y.-C. Pan, H.-X. Zhang, Numerical prediction of marine propeller noise in non-uniform inflow, China Ocean Engineering 27 (1) (2013) 33-42. doi:10.1007/s13344-013-0003-2.

[35] T. P. Lloyd, D. Rijpkema, E. Van Wijngaarden, Implementing the Ffowcs Williams-Hawkings acoustic analogy into a viscous CFD solver, in: 17th Numerical Towing Tank Symposium (NuTTS), 28-30th September, Marstrand, Sweden, 2014.

[36] D.-Q. Li, J. Hallander, R. Karlsson, Study of underwater noise signature from a tanker with a cavitating propeller, in: 17 th Numerical Towing Tank Symposium (NuTTS), 28-30th September, Marstrand, Sweden, 2014.

[37] A. Feymark, M. Liefvendahl, R. E. Bensow, Simulation cases for verification of flow noise prediction in the parameter range of interest for naval applications, in: 17th Numerical Towing Tank Symposium (NuTTS), 28-30th September, Marstrand, Sweden, 2014.

[38] A. S. Lyrintzis, Surface Integral Methods in Computational Aeroacoustics - From the CFD Near-Field to the Acoustic FarField, in: CEAS Workshop "From CFD to CAA", Athens, Greece, 2002, pp. 1-53.
[39] S. Ianniello, R. Muscari, A. D. Mascio, Hydroacoustic characterization of a marine propeller through the acoustic analogy, Sustainable Maritime Transportation and Exploitation of Sea Resources (2012) 991-1000.

[40] A. R. Jamaluddin, G. J. Ball, C. K. Turangan, T. G. Leighton, The collapse of single bubbles and approximation of the farfield acoustic emissions for cavitation induced by shock wave lithotripsy, Journal of Fluid Mechanics 677 (2011) 305-341. doi: $10.1017 / \mathrm{jfm} .2011 .85$.

[41] J. E. Bardina, J. H. Ferziger, W. C. Reynolds, No Title, in: AIAA Fluid and Plasma Dynamics Conference, 14-16 July, Snowmass, 1980.

[42] Y. Zang, R. L. Street, J. R. Koseff, A dynamic mixed subgridscale model and its application to turbulent recirculating flows, Physics of Fluids A: Fluid Dynamics 5 (12) (1993) 3186. doi: $10.1063 / 1.858675$.

[43] M. Germano, U. Piomelli, P. Moin, W. H. Cabot, A dynamic subgridscale eddy viscosity model, Physics of Fluids 3 (7) (1991) 1760-1765.

[44] A. K. Lidtke, S. R. Turnock, V. F. Humphrey, The influence of turbulence modelling techniques on the predicted cavitation behaviour on a NACA0009 foil, in: Numerical Towing Tank Symposium (NuTTS), Marstrand, Sweden, 2014.

[45] E. Foeth, The structure of three-dimensional sheet cavitation, Ph.D. thesis, TU Delft (2008).

[46] M. Hoekstra, T. van Terwisga, E. J. Foeth, SMP11 Workshop - Case 1: DelftFoil, in: Second International Symposium on Marine Propulsors, Hamburg, Germany, 2011.

[47] R. Bensow, M. Liefvendahl, Implicit and explicit subgrid modeling in les applied to a marine propeller, in: 38th Fluid Dynamics Conference and Exhibit, 23 - 26 June, Seattle, Washington, 2008, pp. 1-12.

[48] R. E. Bensow, G. Bark, Implicit LES predictions of the cavitating flow on a propeller, Journal of fluids engineering 132 (4) (2010) 041302. doi:10.1115/1.4001342.

[49] B. Greschner, J. Grilliat, M. C. Jacob, F. Thiele, Measurements and wall modeled LES simulation of trailing edge noise caused by a turbulent boundary layer, International Journal of Aeroacoustics 9 (3) (2010) 329-356. doi:10.1260/1475-472X.9.3.329.

[50] B. Ji, X. Luo, R. E. Arndt, X. Peng, Y. Wu, Large Eddy Simulation and theoretical investigations of the transient cavitating vortical flow structure around a NACA66 hydrofoil, International Journal of Multiphase Flow 68 (2015) 121-134. doi:10.1016/j.ijmultiphaseflow.2014.10.008.

[51] G. Rahier, M. Huet, J. Prieur, Additional terms for the use of Ffowcs Williams and Hawkings surface integrals in turbulent flows, Computers \& Fluids 120 (2015) 158-172. doi:10.1016/j.compfluid.2015.07.014.

[52] S. Gaggero, D. Villa, S. Brizzolara, SMP workshop on cavitation and propeller performances: The experience of the University of Genova on the Potsdam propeller test case, in: International Symposium on Marine Propulsors, no. June, Hamburg, Germany, 2011.

[53] B. Ji, X.-w. Luo, X.-x. Peng, Y.-1. Wu, Three-dimensional large eddy simulation and vorticity analysis of unsteady cavitating flow around a twisted hydrofoil, Journal of Hydrodynamics, Ser. B 25 (4) (2013) 510-519. doi:10.1016/S1001-6058(11)60390$\mathrm{X}$.

[54] D.-Q. Li, M. Grekula, P. Lindell, A modified SST k-omega Turbulence Model to Predict the Steady and Unsteady Sheet Cavitation on 2D and 3D Hydrofoils, in: 7th International Symposium on Cavitation CAV2009, no. 107, August 17-22, Ann Arbor, Michigan, USA, 2009, pp. 1-13.

[55] C. E. Brennen, Cavitation noise, in: Fundamentals of Multiphase Flow, 2nd Edition, Cambridge University Press, Cam- 
bridge, 2009, Ch. 5.3, pp. 109-111.

[56] C. T. Hsiao, G. L. Chahine, Scaling of tip vortex cavitation inception for a marine open propeller, in: 27th Symposium on Naval Hydrodynamics, no. October, Seoul, Korea, 2008, pp. 510 .

[57] J. Matusiak, Pressure and noise induced by a cavitating marine screw propeller, Ph.D. thesis, Helsinki University of Technology (1992).

[58] P. Dupont, Etude de la dynamique d'une poche de cavitation partielle en vue de la prédiction de l'érosion dans les turbomachines hydrauliques, Ph.D. thesis, Ecole Polytechnique Federale de Lausanne (1993).

[59] Y. Ait Bouziad, Physical Modelling of Leading Edge Cavitation: Computational Methodologies and Application To Hydraulic Machinery, Ph.D. thesis, Ecole Polytechnique Federale de Lausanne (2005).

[60] T. Huuva, Large eddy simulation of cavitating and noncavitating flow, Ph.D. thesis, Chalmers University of Technology (2008).

[61] HSVA, Experimental Investigation to Improve Numerical Modeling of Cavitation, Tech. rep., HSVA (2008).

[62] S. L. Ceccio, C. E. Brennen, Observations of the dynamics and acoustics of travelling bubble cavitation, Journal of Fluid Mechanics 233 (-1) (2006) 633. doi:10.1017/S0022112091000630.

[63] C. E. Brennen, T. Colonius, F. D' Auria, Computing shock waves in cloud cavitation, in: Third International Symposium on Cavitation, Grenoble, France, 1998, pp. 287-294.

URL http://authors.library.caltech.edu/195/

[64] T. G. Leighton, C. K. Turangan, a. R. Jamaluddin, G. J. Ball, P. R. White, Prediction of far-field acoustic emissions from cavitation clouds during shock wave lithotripsy for development of a clinical device, Proceedings of the Royal Society A: Mathematical, Physical and Engineering Sciences 469 (2150) (2012) 20120538-20120538. doi:10.1098/rspa.2012.0538.

[65] R. Raju, S. Singh, H. Chao-tsung, C. Georges, Study of pressure wave propagation in a two-phase bubbly mixture, in: ASME International Mechanical Engineering COngress \& Exposition, Vancouver, British Columbia, Canada, 2010, pp. 1-36.

[66] T. B. Benjamin, A. T. Elis, The collapse of cavitation bubbles and the pressures thereby produced against solid boundaries, Philosophical Transactions of the Royal Society of Londong 260 (1110).

[67] S. Ianniello, R. Muscari, A. Mascio, Ship underwater noise assessment by the acoustic analogy. Part I: nonlinear analysis of a marine propeller in a uniform flow, Journal of Marine Science and Technology 18 (4) (2013) 547-570. doi:10.1007/s00773013-0227-0.

[68] S. Sinayoko, M. C. M. Wright, R. D. Sandberg, A generalised Ffowcs-Williams and Hawkings formulation applied to flow simulations with vortical outflow, in: 22nd International Congress on Sound and Vibration, 12-16 July, Florence, Italy, 2015.

[69] B. Godderidge, S. R. Turnock, C. Earl, M. Tan, The effect of fluid compressibility on the simulation of sloshing impacts, Ocean Engineering 36 (8) (2009) 578-587. doi:10.1016/j.oceaneng.2009.02.004.

[70] T. Ménard, S. Tanguy, A. Berlemont, Coupling level set/VOF/ghost fluid methods: Validation and application to 3D simulation of the primary break-up of a liquid jet, International Journal of Multiphase Flow 33 (5) (2007) 510-524. doi:10.1016/j.ijmultiphaseflow.2006.11.001.

[71] X. Lv, Q. Zou, Y. Zhao, D. Reeve, A novel coupled level set and volume of fluid method for sharp interface capturing on 3D tetrahedral grids, Journal of Computational Physics 229 (7) (2010) 2573-2604. doi:10.1016/j.jcp.2009.12.005. 